\title{
Review
}

\section{Applications of Smart Helmet in Applied Sciences: A Systematic Review}

\author{
Yosoon Choi * ${ }^{*}$ and Yeanjae Kim \\ Department of Energy Resources Engineering, Pukyong National University, Busan 48513, Korea; \\ yeanbean22@naver.com \\ * Correspondence: energy@pknu.ac.kr; Tel.: +82-51-629-6562; Fax: +82-51-629-6553
}

check for updates

Citation: Choi, Y.; Kim, Y.

Applications of Smart Helmet in

Applied Sciences: A Systematic

Review. Appl. Sci. 2021, 11, 5039.

https://doi.org/10.3390/

app11115039

Academic Editor: Juan Francisco De Paz Santana

Received: 25 February 2021

Accepted: 27 May 2021

Published: 29 May 2021

Publisher's Note: MDPI stays neutral with regard to jurisdictional claims in published maps and institutional affiliations.

Copyright: (c) 2021 by the authors. Licensee MDPI, Basel, Switzerland. This article is an open access article distributed under the terms and conditions of the Creative Commons Attribution (CC BY) license (https:// creativecommons.org/licenses/by/ $4.0 /)$.

\begin{abstract}
A smart helmet is a wearable device that has attracted attention in various fields, especially in applied sciences, where extensive studies have been conducted in the past decade. In this study, the current status and trends of smart helmet research were systematically reviewed. Five research questions were set to investigate the research status of smart helmets according to the year and application field, as well as the trend of smart helmet development in terms of types of sensors, microcontrollers, and wireless communication technology. A total of 103 academic research articles published in the past 11 years (2009-2020) were analyzed to address the research questions. The results showed that the number of smart helmet applications reported in literature has been increasing rapidly since 2018. The applications have focused mostly on ensuring the safety of motorcyclists. A single-board-based modular concept unit, such as the Arduino board, and sensor for monitoring human health have been used the most for developing smart helmets. Approximately $85 \%$ of smart helmets have been developed to date using wireless communication technology to transmit data obtained from smart helmets to other smart devices or cloud servers.
\end{abstract}

Keywords: wearable device; smart helmet; sensor; microcontroller; wireless communication technology

\section{Introduction}

A wearable (electronic) device is a smart electronic device that can be planted on the body or worn with accessories. Since Google recently launched its head-mounted display, wearable devices have garnered a significant amount of attention [1]. Wearable devices have been able to take an important position in the home appliance market in a short period and are considered a new means of meeting the requirements of many industries. For example, the construction industry has studied the use of wearable devices in the workplace for health and safety management by close detection and physiological monitoring of construction workers [2]. The logistics industry has begun using wearable barcode scanner gloves to simplify work that does not involve hand use [3]. Some insurance companies encourage healthy eating habits and use wearable devices to improve the health of corporate workers [4].

There are different types of smart wearable devices, such as helmets, watches, glasses, contact lenses, textiles, fabrics, headbands, beanies, caps, rings, bracelets, and earrings [5]. Among these, this study focused on smart helmets. Smart helmets include multiple electronic devices and sensors that help users gather real-time data and assist them in reducing operational risks and improving safety in the long run. The global smart helmet market size was valued at USD 372.4 million in 2018 and is expected to expand at a compound annual growth rate of $18.6 \%$ from 2019 to 2025 [6].

Research on smart helmet applications is being conducted in various fields to improve safety and efficiency of motorcyclists [7] and workers [8,9]. For example, Singh et al. [7] studied the application of smart helmets to detect portholes and collect air quality data 
on roads. Wang et al. [8] detected stair fall by analyzing changes in weight support and pressure center using a smart helmet, and Mohammed et al. [9] used smart helmets to prevent the spread of coronavirus by measuring the body temperature and personal information of pedestrians. Like some of the examples mentioned earlier, many studies related to the development and application of smart helmets have been reported in the literature [10-109]; however, no systematic review has been conducted to analyze the current status and trends of smart helmet research.

The purpose of this study was to review the current status and trends of smart helmet research systematically. The current status of smart helmet research by year and application field were investigated by analyzing 103 academic research articles published in the past 11 years (2009-2020). The types of sensors, microcontrollers, and wireless communication technology were analyzed to identify the trends in smart helmet studies.

\section{Research Methodology}

In this study, the systematic review was designed using five steps as follows.

(1) Research questions were defined to conduct a systematic review of smart helmets.

(2) A search method was designed to identify academic research articles related to the research questions.

(3) Selection criteria were set to distinguish between articles related to the research questions and those not related to them.

(4) The selected articles were analyzed, and the results were integrated.

(5) The results were summarized and reported to provide answers to the research questions.

\subsection{Definition of Research Questions}

Five research questions (RQs) were defined to review the current status and trends of smart helmet research.

- RQ1: How is the trend of publications related to smart helmet research by year and source?

- $\quad$ RQ2: In which fields has the smart helmet research been mainly conducted?

- RQ3: What kinds of sensor have been mainly used for smart helmet applications?

- RQ4: What types of microcontroller have been primarily used to develop smart helmets?

- RQ5: What kinds of wireless communication technology have been mainly used to connect smart helmet with other devices?

\subsection{Literature Search and Selection}

Related academic articles were initially searched and identified using the Web of Science, Scopus, and Google Scholar databases. The initial search keyword "smart helmet" was used, which provided numerous articles. Another search was conducted using such keywords as "Sensor", "IOT," "Arduino," and "Raspberry Pi." A total of 125 articles were found. Four selection criteria were established and used for the 125 articles. The selection criteria excluded the articles when (i) the article was duplicate, (ii) the article was not an academic research report, (iii) the article was not a full-text article, and (iv) information about microcontrollers was not clearly presented. Of these 125 articles, one article was excluded as a result of reviewing for duplicate articles. Six articles that were not academic research reports were excluded. Seven articles that were not full-text articles were excluded, and eight articles were excluded in which information about microcontrollers was not clearly presented. The final selection included 103 articles that met the selection criteria. For these 103 articles, a systematic review was conducted according to each research question. Figure 1 presents a flow chart of the selection criteria. 


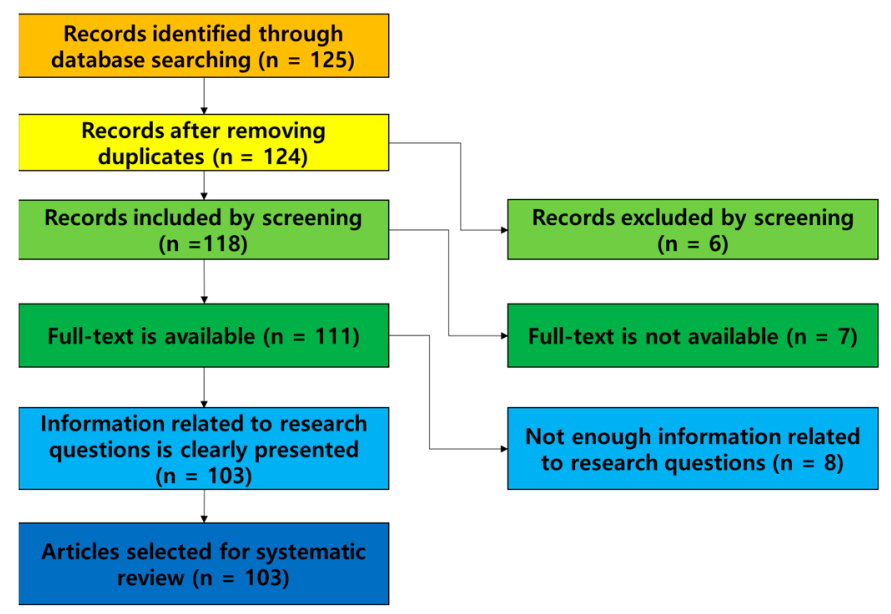

Figure 1. Flow chart of selection criteria.

\subsection{Data Analysis and Outline of Results}

The 103 articles obtained from literature selection were classified to separate contents of the articles and facilitate interpretation of the data. This analysis was conducted for each individual publication by considering the RQs and outline of the results presented in Table 1.

Table 1. Outline of results for each research question.

\begin{tabular}{|c|c|c|c|c|c|}
\hline Year & & plication Field & Sensor & Microcontroller & $\begin{array}{c}\text { Wireless } \\
\text { Communication } \\
\text { Technology }\end{array}$ \\
\hline $\begin{array}{l}2009 \\
2010 \\
2011 \\
2012 \\
2013 \\
2014 \\
2015 \\
2016 \\
2017 \\
2018 \\
2019 \\
2020\end{array}$ & Motorcyclist & $\begin{array}{l}\text { - Start control } \\
\text { - Accident prevention } \\
\text { - Rescue request } \\
\text { - Convenience improvement } \\
\text { - Mining } \\
\text { - Construction } \\
\text { - Petrochemistry } \\
\text { - Disaster prevention } \\
\text { - Medical treatment } \\
\text { - General }\end{array}$ & $\begin{array}{l}\text { - Human health } \\
\text { - Environment } \\
\text { - Machine }\end{array}$ & $\begin{array}{l}\text { - Chip modular } \\
\text { concept unit } \\
\text { (MCU) } \\
\text { - Single-board } \\
\text { MCU } \\
\text { - Single-board } \\
\text { computer } \\
\text { - Smart device }\end{array}$ & $\begin{array}{l}\text { - Bluetooth } \\
\text { - RF } \\
\text { - Zigbee } \\
\text { - WiFi }\end{array}$ \\
\hline
\end{tabular}

\section{Results}

\subsection{Current Status of Publications Related to Smart Helmet Research by Year and Source}

The number of publications on smart helmets during the past 11 years is shown in Figure 2. In the first seven years (2009-2015), there were limited articles, with an average of 1.28 articles per year. In the next two years (2016 and 2017), the average number of publications increased to 8.5 . In 2018, the number of publications more than doubled from the previous year's average, surging to 21 publications. In 2019, there were 27 publications, and, in 2020, the number of publications was 29, making it the highest number for a year overall. The average number of publications in the last three years (2018-2020) was 25.6, which is nearly three times higher than the average number of publications in 2016 and 2017. Therefore, research on smart helmets has been extremely active recently. Additionally, the sources of publications were academic journals (55.3\%) and conference proceedings $(44.7 \%)$. 


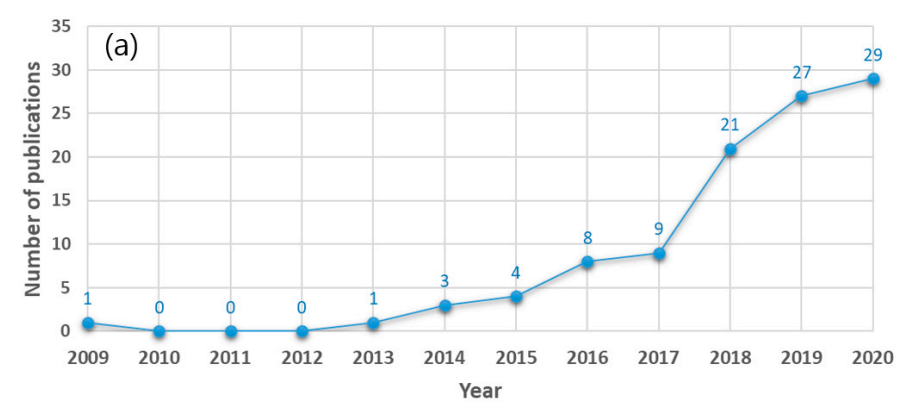

(b)

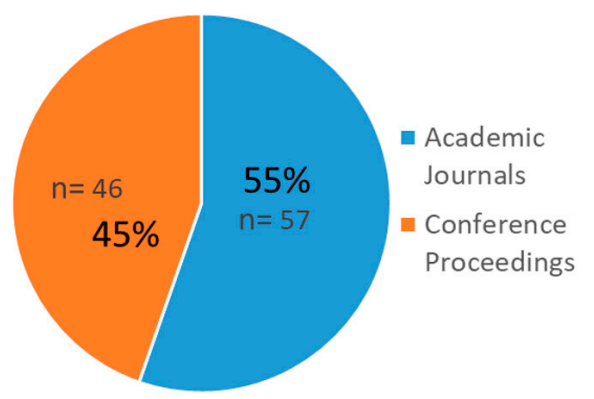

Figure 2. Number of publications related to smart helmet research by (a) year and (b) source of publications.

\subsection{Application Fields of Smart Helmet Research}

The current status of smart helmet studies in each application field was analyzed to determine which fields were studying and utilizing smart helmets the most. The smart helmet applications were organized in two stages to observe the classification at a glance. In the first stage, the studies were classified into two categories (i.e., motorcyclists and workers) according to users of smart helmets. In the second stage, detailed application fields were added below the first stage, as shown in Figure 3. Figure 4 shows the cumulative number of publications on smart helmets during the past 11 years in the first stage of the classification criteria. As shown, studies on smart helmets for motorcyclists and workers have been increasing every year; however, the rate of increase is relatively faster for motorcyclists. This indicates that research on smart helmets for workers must be accelerated.

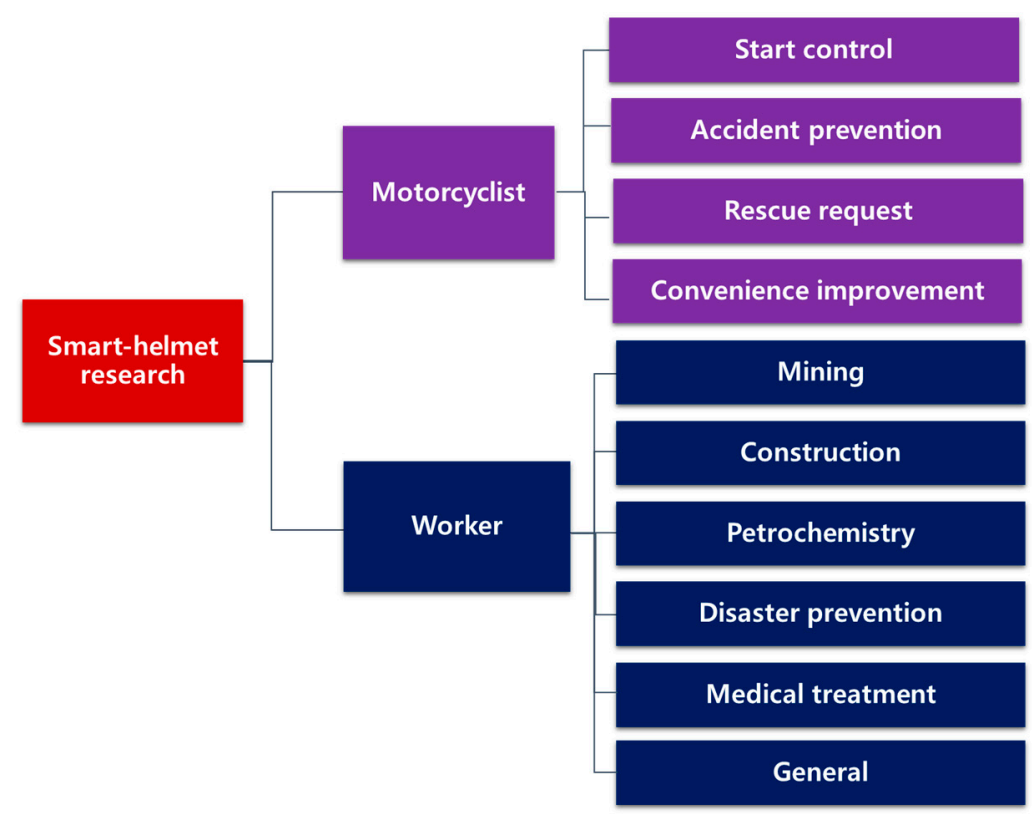

Figure 3. Classification of application fields in smart helmet studies. 


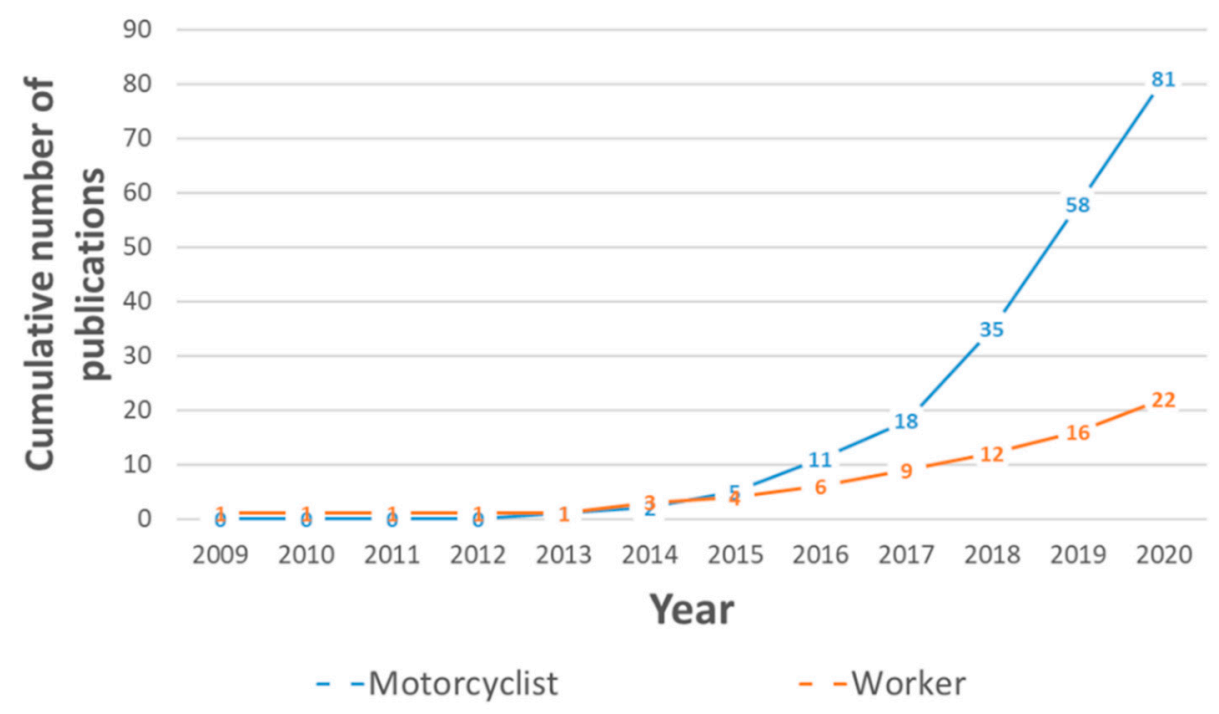

Figure 4. Cumulative number of publications by year in bike, occupational safety, disaster prevention, and medicine fields.

The applications of smart helmets for motorcyclists consist of 81 studies that account for the largest number of publications [7,10-80]. These applications can be classified into four subcategories: start control, accident prevention, rescue request, and convenience improvement. Some studies fall under more than one subcategory when the developed smart helmet is used for various purposes by motorcyclists. Figure 5 shows the percentage of studies according to the subcategory. Note that most smart helmets for motorcyclists have been developed for the start control of motorcycles $(42.3 \%)$ and rescue requests in the case of an accident (39.2\%).

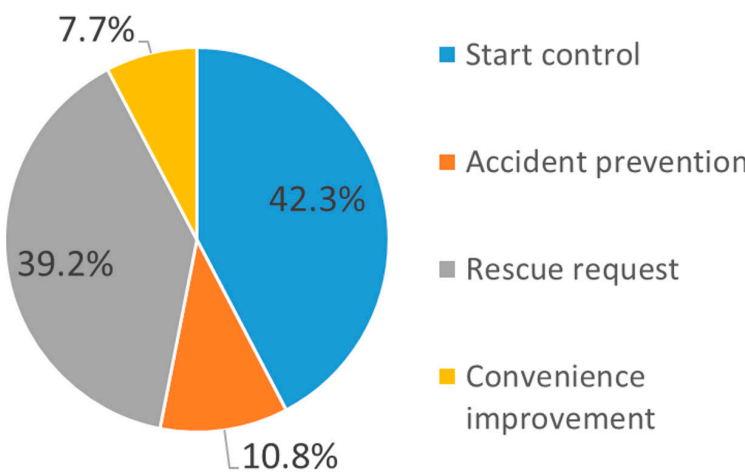

Figure 5. Percentage of smart helmet studies for motorcyclists by sub-category.

Table 2 summarizes the smart helmet applications reported in 81 studies for start control, accident prevention, rescue requests, and convenience improvement of motorcyclists. For the start control, the smart helmet checks whether the motorcyclist is wearing the helmet and for alcohol consumption. If any of the conditions are not met, the smart helmet controls the motorcycle to not start. For accident prevention, smart helmets are responsible for detecting drowsiness, speed and blinking, and providing warning alerts to prevent accidents. In the rescue request, the smart helmets detect a traffic accident and make a rescue request through text or phone calls in the event of an accident. Finally, for improving convenience of motorcyclists, the smart helmet provides them with several functions, including music play, phone calls, audio, and navigation. 
Table 2. Summary of smart helmet applications for start control, accident prevention, rescue request, and convenience improvement of motorcyclists (MCU: modular concept unit).

\begin{tabular}{|c|c|c|c|c|c|c|}
\hline Reference & Year & $\begin{array}{l}\text { Feature and High-Level } \\
\text { Functionality }\end{array}$ & $\begin{array}{l}\text { Application } \\
\text { (Sub-Category) }\end{array}$ & Sensor & Microcontroller & $\begin{array}{l}\text { Wireless } \\
\text { Technology }\end{array}$ \\
\hline Singh et al. [7] & 2019 & $\begin{array}{l}\text { Report potholes, collect air } \\
\text { pollution data, and send it to a } \\
\text { cloud server for analysis }\end{array}$ & $\begin{array}{l}\text { Accident } \\
\text { prevention }\end{array}$ & $\begin{array}{l}\text { Porthole detection; } \\
\text { Harmful gas } \\
\text { detection }\end{array}$ & $\begin{array}{l}\text { Smart device } \\
\text { (Smartphone) }\end{array}$ & $\mathrm{RF}$ \\
\hline Rasli et al. [10] & 2013 & $\begin{array}{l}\text { Starts only when the helmet } \\
\text { buckle is engaged, and warning is } \\
\text { sent through the LED when } \\
\text { over-speeding }\end{array}$ & $\begin{array}{l}\text { Start control; } \\
\text { Accident } \\
\text { prevention }\end{array}$ & $\begin{array}{l}\text { Helmet wear } \\
\text { detection; } \\
\text { Speed detection }\end{array}$ & $\begin{array}{l}\text { Chip MCU } \\
\text { (PIC) }\end{array}$ & $\mathrm{RF}$ \\
\hline $\begin{array}{l}\text { Vijayan et al. } \\
\text { [11] }\end{array}$ & 2014 & $\begin{array}{l}\text { Start-up management and } \\
\text { accident prevention by collecting } \\
\text { data on alcohol consumption and } \\
\text { wearing a helmet }\end{array}$ & Start control & $\begin{array}{l}\text { Alcohol detection; } \\
\text { Helmet wear } \\
\text { detection }\end{array}$ & $\begin{array}{l}\text { Chip MCU } \\
\text { (PIC) }\end{array}$ & RF \\
\hline $\begin{array}{l}\text { Agarwal et al. } \\
\text { [12] }\end{array}$ & 2015 & $\begin{array}{l}\text { Startup management by detecting } \\
\text { whether a helmet is worn }\end{array}$ & Start control & $\begin{array}{l}\text { Helmet wear } \\
\text { detection }\end{array}$ & $\begin{array}{l}\text { Chip MCU } \\
\text { (Intel8051) }\end{array}$ & $\mathrm{RF}$ \\
\hline $\begin{array}{l}\text { Gautam et al. } \\
\text { [13] }\end{array}$ & 2015 & $\begin{array}{l}\text { Detects whether a helmet is worn } \\
\text { or alcohol is consumed, and when } \\
\text { both conditions are met, the } \\
\text { engine starts up. Else, a text } \\
\text { message is sent to a designated } \\
\text { number when one of the } \\
\text { conditions is not met or when an } \\
\text { accident is detected }\end{array}$ & $\begin{array}{l}\text { Start control; } \\
\text { Rescue request }\end{array}$ & $\begin{array}{l}\text { Helmet wear } \\
\text { detection; Alcohol } \\
\text { detection; Accident } \\
\text { detection }\end{array}$ & $\begin{array}{l}\text { Chip MCU } \\
\text { (AT89S52) }\end{array}$ & $\mathrm{RF}$ \\
\hline $\begin{array}{l}\text { Melcher et al. } \\
\text { [14] }\end{array}$ & 2015 & $\begin{array}{l}\text { Collect vital signs to check the } \\
\text { driver's condition and request } \\
\text { rescue by phone or text in case of } \\
\text { an emergency }\end{array}$ & Rescue request & Vital Sign detection & $\begin{array}{l}\text { Smart device } \\
\text { (Smartphone) }\end{array}$ & Bluetooth \\
\hline $\begin{array}{l}\text { Chandran et al. } \\
\text { [15] }\end{array}$ & 2016 & $\begin{array}{l}\text { Detects an accident and connects } \\
\text { to the cloud server via WiFi; } \\
\text { requests rescue from the } \\
\text { emergency contact network }\end{array}$ & Rescue request & Accident detection & $\begin{array}{l}\text { Chip MCU } \\
(\text { CC3200) }\end{array}$ & WiFi \\
\hline $\begin{array}{l}\text { Magno et al. } \\
{[16]}\end{array}$ & 2016 & $\begin{array}{l}\text { Situational awareness, blinking, } \\
\text { drinking, and temperature data } \\
\text { are collected to ensure driver } \\
\text { safety; low power modules allow } \\
\text { the system to run for a long time }\end{array}$ & $\begin{array}{l}\text { Accident } \\
\text { prevention }\end{array}$ & $\begin{array}{l}\text { Eye blink detection; } \\
\text { Alcohol detection; } \\
\text { Temperature } \\
\text { detection }\end{array}$ & $\begin{array}{l}\text { Chip MCU } \\
\text { (MSP432) }\end{array}$ & $\mathrm{RF}$ \\
\hline $\begin{array}{l}\text { Nikharge et al. } \\
\text { [17] }\end{array}$ & 2016 & $\begin{array}{l}\text { Raspberry Pi image processing } \\
\text { detects whether a helmet is worn }\end{array}$ & Start control & $\begin{array}{l}\text { Helmet wear } \\
\text { detection }\end{array}$ & $\begin{array}{l}\text { Single board } \\
\text { computer } \\
\text { (Raspberry Pi) }\end{array}$ & \\
\hline $\begin{array}{l}\text { Jadhawar et al. } \\
\text { [18] }\end{array}$ & 2016 & $\begin{array}{l}\text { Detects whether a helmet is worn } \\
\text { and drinking alcohol, and when } \\
\text { both conditions are satisfied, the } \\
\text { engine is started, and an accident } \\
\text { is detected through a fall detection } \\
\text { and a text message is sent to } \\
\text { request rescue }\end{array}$ & $\begin{array}{l}\text { Start control; } \\
\text { Rescue request }\end{array}$ & $\begin{array}{l}\text { Helmet wear } \\
\text { detection; Alcohol } \\
\text { detection; Accident } \\
\text { detection }\end{array}$ & $\begin{array}{l}\text { Chip MCU } \\
\text { (ATMEGA32) }\end{array}$ & \\
\hline Kim et al. [19] & 2016 & $\begin{array}{l}\text { Rescue request by detecting an } \\
\text { accident and sending a text } \\
\text { message to the registered number }\end{array}$ & Rescue request & Accident detection & $\begin{array}{l}\text { Single board } \\
\text { MCU } \\
\text { (Arduino) }\end{array}$ & Bluetooth \\
\hline $\begin{array}{l}\text { Kumar et al. } \\
\text { [20] }\end{array}$ & 2016 & $\begin{array}{l}\text { Detects whether a helmet is worn } \\
\text { and alcohol is consumed. When } \\
\text { both conditions are met, the } \\
\text { engine is started, an accident is } \\
\text { detected, and connection is made } \\
\text { to the cloud server to request } \\
\text { rescue through a mobile } \\
\text { phone app. }\end{array}$ & $\begin{array}{l}\text { Start control; } \\
\text { Rescue request }\end{array}$ & $\begin{array}{l}\text { Alcohol detection; } \\
\text { Accident detection; } \\
\text { Helmet wear } \\
\text { detection }\end{array}$ & $\begin{array}{l}\text { Single board } \\
\text { MCU } \\
\text { (Arduino) }\end{array}$ & WiFi \\
\hline
\end{tabular}


Table 2. Cont.

\begin{tabular}{|c|c|c|c|c|c|c|}
\hline Reference & Year & $\begin{array}{l}\text { Feature and High-Level } \\
\text { Functionality }\end{array}$ & $\begin{array}{c}\text { Application } \\
\text { (Sub-Category) }\end{array}$ & Sensor & Microcontroller & $\begin{array}{l}\text { Wireless } \\
\text { Technology }\end{array}$ \\
\hline $\begin{array}{l}\text { Vashisth et al. } \\
\text { [21] }\end{array}$ & 2017 & $\begin{array}{l}\text { Detects whether the helmet is } \\
\text { worn and if the driver is speeding } \\
\text { in an inebriated state; if so, a text } \\
\text { message is sent to request rescue } \\
\text { in the event of an accident }\end{array}$ & $\begin{array}{l}\text { Start control; } \\
\text { Accident } \\
\text { prevention; } \\
\text { Rescue request }\end{array}$ & $\begin{array}{l}\text { Helmet wear } \\
\text { detection; Speed } \\
\text { detection; Alcohol } \\
\text { detection }\end{array}$ & $\begin{array}{l}\text { Single board } \\
\text { MCU } \\
\text { (Arduino) }\end{array}$ & RF \\
\hline $\begin{array}{l}\text { Varade et al. } \\
\text { [22] }\end{array}$ & 2017 & $\begin{array}{l}\text { Detects whether the helmet is } \\
\text { worn, sends the acceleration value } \\
\text { to the cloud server for analysis, } \\
\text { and requests rescue via text in case } \\
\text { of an accident }\end{array}$ & $\begin{array}{l}\text { Start control; } \\
\text { Rescue request }\end{array}$ & $\begin{array}{l}\text { Helmet wear } \\
\text { detection }\end{array}$ & $\begin{array}{l}\text { Chip MCU } \\
\text { (ATMEGA16) }\end{array}$ & Bluetooth \\
\hline $\begin{array}{l}\text { Rajput et al. } \\
\text { [23] }\end{array}$ & 2017 & $\begin{array}{l}\text { Detects alcohol consumption, } \\
\text { manages the start-up through } \\
\text { fingerprint recognition, and sends } \\
\text { a text message to the vehicle } \\
\text { owner when the vehicle is stolen. }\end{array}$ & Start control & Alcohol detection & $\begin{array}{l}\text { Single board } \\
\text { MCU } \\
\text { (Arduino) }\end{array}$ & \\
\hline Patel et al. [24] & 2017 & $\begin{array}{l}\text { Start-up management based on } \\
\text { whether a helmet is worn, and an } \\
\text { accident is detected and a text } \\
\text { message is sent. }\end{array}$ & $\begin{array}{l}\text { Start control; } \\
\text { Rescue request }\end{array}$ & $\begin{array}{l}\text { Helmet wear } \\
\text { detection; Accident } \\
\text { detection }\end{array}$ & $\begin{array}{l}\text { Single board } \\
\text { MCU } \\
\text { (Arduino) }\end{array}$ & Bluetooth \\
\hline $\begin{array}{l}\text { Muthiah et al. } \\
\text { [25] }\end{array}$ & 2017 & $\begin{array}{l}\text { Introduction of autonomous } \\
\text { headlight technology }\end{array}$ & $\begin{array}{l}\text { Convenience } \\
\text { improvement }\end{array}$ & & $\begin{array}{l}\text { Single board } \\
\text { MCU } \\
\text { (Arduino) }\end{array}$ & RF \\
\hline $\begin{array}{l}\text { Ahuja and } \\
\text { Bhavsar [26] }\end{array}$ & 2018 & $\begin{array}{l}\text { Vibration and shake sensors detect } \\
\text { accidents and send voice messages } \\
\text { and texts when they occur }\end{array}$ & Rescue request & Accident detection & $\begin{array}{l}\text { Single board } \\
\text { MCU } \\
\text { (Arduino) }\end{array}$ & \\
\hline $\begin{array}{l}\text { Godwani et al. } \\
\text { [27] }\end{array}$ & 2018 & $\begin{array}{l}\text { Detects people's actions and } \\
\text { shocks and sends text messages to } \\
\text { family and acquaintances in case } \\
\text { of an accident }\end{array}$ & Rescue request & Accident detection & $\begin{array}{l}\text { Single board } \\
\text { computer } \\
\text { (Raspberry Pi) }\end{array}$ & WiFi \\
\hline $\begin{array}{l}\text { Parameshwari } \\
\text { et al. [28] }\end{array}$ & 2018 & $\begin{array}{l}\text { Detects whether a helmet is worn } \\
\text { or drinking alcohol, and when } \\
\text { both conditions are met, the } \\
\text { engine starts up and a text } \\
\text { message is sent in case of an } \\
\text { accident. }\end{array}$ & $\begin{array}{l}\text { Start control; } \\
\text { Rescue request }\end{array}$ & $\begin{array}{l}\text { Helmet wear } \\
\text { detection; Alcohol } \\
\text { detection; Accident } \\
\text { detection }\end{array}$ & $\begin{array}{l}\text { Single board } \\
\mathrm{MCU} \\
\text { (Arduino) }\end{array}$ & Bluetooth \\
\hline $\begin{array}{l}\text { Biswas et al. } \\
\text { [29] }\end{array}$ & 2018 & $\begin{array}{l}\text { Detects an incident and sends an } \\
\text { alert to nearby hospitals and other } \\
\text { authorities by an app on your } \\
\text { mobile phone connected to Google } \\
\text { Firebase, which can be stopped via } \\
\text { a PIN number }\end{array}$ & Rescue request & Accident detection & $\begin{array}{l}\text { Single board } \\
\text { computer } \\
\text { (Raspberry Pi) }\end{array}$ & Bluetooth \\
\hline $\begin{array}{l}\text { Budiman et al. } \\
\text { [30] }\end{array}$ & 2018 & $\begin{array}{l}\text { Detect whether a helmet is worn } \\
\text { and over speeding }\end{array}$ & $\begin{array}{l}\text { Start control; } \\
\text { Accident } \\
\text { prevention }\end{array}$ & $\begin{array}{l}\text { Helmet wear } \\
\text { detection; Speed } \\
\text { detection }\end{array}$ & $\begin{array}{l}\text { Single board } \\
\text { MCU } \\
\text { (Arduino) }\end{array}$ & \\
\hline $\begin{array}{l}\text { Uniyal et al. } \\
\text { [31] }\end{array}$ & 2018 & $\begin{array}{l}\text { Collects helmet wearing status, } \\
\text { location, speeding, and collision } \\
\text { data; sends it to the cloud server; } \\
\text { and requests rescue from nearby } \\
\text { emergency facilities in case of an } \\
\text { accident }\end{array}$ & $\begin{array}{l}\text { Start control; } \\
\text { Accident } \\
\text { prevention; } \\
\text { Rescue request }\end{array}$ & $\begin{array}{l}\text { Helmet wear } \\
\text { detection; Speed } \\
\text { detection; Accident } \\
\text { detection; Location } \\
\text { detection }\end{array}$ & $\begin{array}{l}\text { Single board } \\
\mathrm{MCU} \\
\text { (Arduino) }\end{array}$ & RF \\
\hline $\begin{array}{l}\text { Paulchamy } \\
\text { et al. [32] }\end{array}$ & 2018 & $\begin{array}{l}\text { Detects whether a helmet is worn } \\
\text { or alcohol is consumed; starts the } \\
\text { engine when both conditions are } \\
\text { met; collects location information; } \\
\text { and sends text messages in case of } \\
\text { an accident }\end{array}$ & $\begin{array}{l}\text { Start control; } \\
\text { Rescue request }\end{array}$ & $\begin{array}{l}\text { Helmet wear } \\
\text { detection; Alcohol } \\
\text { detection; Accident } \\
\text { detection }\end{array}$ & $\begin{array}{l}\text { Single board } \\
\text { MCU } \\
\text { (Arduino) }\end{array}$ & RF \\
\hline $\begin{array}{l}\text { Souza and } \\
\text { Maliyackal [33] }\end{array}$ & 2018 & $\begin{array}{l}\text { Start management by detecting } \\
\text { whether a helmet is worn }\end{array}$ & Start control & $\begin{array}{l}\text { Helmet wear } \\
\text { detection }\end{array}$ & $\begin{array}{l}\text { Single board } \\
\text { MCU } \\
\text { (Arduino) }\end{array}$ & $\mathrm{RF}$ \\
\hline
\end{tabular}


Table 2. Cont.

\begin{tabular}{|c|c|c|c|c|c|c|}
\hline Reference & Year & $\begin{array}{l}\text { Feature and High-Level } \\
\text { Functionality }\end{array}$ & $\begin{array}{c}\text { Application } \\
\text { (Sub-Category) }\end{array}$ & Sensor & Microcontroller & $\begin{array}{l}\text { Wireless } \\
\text { Technology }\end{array}$ \\
\hline $\begin{array}{l}\text { Nanda et al. } \\
\text { [34] }\end{array}$ & 2018 & $\begin{array}{l}\text { Collecting data, such as alcohol } \\
\text { consumption, driver's license, } \\
\text { blinking eyes, vibration, collision } \\
\text { red light, etc. }\end{array}$ & $\begin{array}{l}\text { Start control; } \\
\text { Accident } \\
\text { prevention; } \\
\text { Rescue request }\end{array}$ & $\begin{array}{l}\text { Alcohol detection; } \\
\text { Driver's license } \\
\text { detection; Eye blink } \\
\text { detection; Accident } \\
\text { detection }\end{array}$ & $\begin{array}{l}\text { Single board } \\
\text { computer } \\
\text { (Raspberry Pi) }\end{array}$ & $\mathrm{RF}$ \\
\hline Deva et al. [35] & 2018 & $\begin{array}{l}\text { Start management by detecting } \\
\text { whether a helmet is worn }\end{array}$ & Start control & $\begin{array}{l}\text { Helmet wear } \\
\text { detection }\end{array}$ & $\begin{array}{l}\text { Chip MCU } \\
\text { (ATMEGA16) }\end{array}$ & Bluetooth \\
\hline $\begin{array}{l}\text { Sumamah et al. } \\
\text { [36] }\end{array}$ & 2018 & $\begin{array}{l}\text { Detects an accident and send a text } \\
\text { message in the event of an } \\
\text { accident }\end{array}$ & Rescue request & Accident detection & $\begin{array}{l}\text { Single board } \\
\text { MCU } \\
\text { (Arduino) }\end{array}$ & \\
\hline $\begin{array}{l}\text { Shabbeer and } \\
\text { Meleet [37] }\end{array}$ & 2018 & $\begin{array}{l}\text { Detects an accident, marks the } \\
\text { location on the map, and sends a } \\
\text { text message }\end{array}$ & Rescue request & Accident detection & $\begin{array}{l}\text { Single board } \\
\text { MCU } \\
\text { (Arduino) }\end{array}$ & WiFi \\
\hline $\begin{array}{l}\text { Gudavalli et al. } \\
\text { [38] }\end{array}$ & 2018 & $\begin{array}{l}\text { By recognizing the RFID tag, it } \\
\text { locks and unlocks to ensure } \\
\text { security, and detects whether a } \\
\text { helmet is worn to manage the boot }\end{array}$ & Start control & $\begin{array}{l}\text { Helmet wear } \\
\text { detection }\end{array}$ & $\begin{array}{l}\text { Single board } \\
\text { MCU } \\
\text { (Arduino) }\end{array}$ & $\mathrm{RF}$ \\
\hline $\begin{array}{l}\text { Tapadar et al. } \\
\text { [39] }\end{array}$ & 2018 & $\begin{array}{l}\text { Detects alcohol consumption, } \\
\text { helmet-wearing status, and } \\
\text { accidents; collects data; learns it by } \\
\text { machine learning with the SVM } \\
\text { model; and then measures the } \\
\text { accuracy }\end{array}$ & $\begin{array}{l}\text { Start control; } \\
\text { Rescue request }\end{array}$ & $\begin{array}{l}\text { Alcohol detection; } \\
\text { Helmet wear } \\
\text { detection; Accident } \\
\text { detection }\end{array}$ & $\begin{array}{l}\text { Single board } \\
\text { MCU } \\
\text { (Arduino) }\end{array}$ & Bluetooth \\
\hline $\begin{array}{l}\text { Premalatha and } \\
\text { Nandhini [40] }\end{array}$ & 2018 & $\begin{array}{l}\text { Detects drinking and accidents } \\
\text { and sends text messages in case of } \\
\text { accidents }\end{array}$ & $\begin{array}{l}\text { Start control; } \\
\text { Rescue request }\end{array}$ & $\begin{array}{l}\text { Alcohol detection; } \\
\text { Accident detection }\end{array}$ & $\begin{array}{l}\text { Chip MCU } \\
\text { (PIC) }\end{array}$ & $\mathrm{RF}$ \\
\hline $\begin{array}{l}\text { Dhulavvagol } \\
\text { et al. [41] }\end{array}$ & 2018 & $\begin{array}{l}\text { Detects drinking and accidents } \\
\text { and sends text messages in case of } \\
\text { accidents }\end{array}$ & $\begin{array}{l}\text { Start control; } \\
\text { Rescue request }\end{array}$ & $\begin{array}{l}\text { Alcohol detection; } \\
\text { Accident detection }\end{array}$ & $\begin{array}{l}\text { Single board } \\
\text { MCU } \\
\text { (Arduino) }\end{array}$ & WiFi \\
\hline $\begin{array}{l}\text { Joshi and Joshi } \\
\text { [42] }\end{array}$ & 2019 & $\begin{array}{l}\text { Start-up management by } \\
\text { collecting data on drinking, } \\
\text { speeding, and wearing a helmet }\end{array}$ & $\begin{array}{l}\text { Start control; } \\
\text { Accident } \\
\text { prevention }\end{array}$ & $\begin{array}{l}\text { Alcohol detection; } \\
\text { Speed detection; } \\
\text { Helmet wear } \\
\text { detection }\end{array}$ & $\begin{array}{l}\text { Chip MCU } \\
\text { (PIC) }\end{array}$ & $\mathrm{RF}$ \\
\hline Namayala [43] & 2019 & $\begin{array}{l}\text { Start-up management by } \\
\text { collecting data on drinking alcohol } \\
\text { and wearing a helmet }\end{array}$ & Start control & $\begin{array}{l}\text { Alcohol detection; } \\
\text { Helmet wear } \\
\text { detection }\end{array}$ & $\begin{array}{l}\text { Single board } \\
\text { MCU } \\
\text { (Arduino) }\end{array}$ & $\mathrm{RF}$ \\
\hline Wong et al. [44] & 2019 & $\begin{array}{l}\text { Collect and analyze data on } \\
\text { driver's head movement }\end{array}$ & $\begin{array}{l}\text { Accident } \\
\text { prevention }\end{array}$ & $\begin{array}{l}\text { Head motion } \\
\text { detection }\end{array}$ & $\begin{array}{l}\text { Smart device } \\
\text { (Smartphone) }\end{array}$ & \\
\hline $\begin{array}{l}\text { Kinage and } \\
\text { Patil [45] }\end{array}$ & 2019 & $\begin{array}{l}\text { Drinking and speeding are } \\
\text { prevented, and drowsiness } \\
\text { detection programs are applied } \\
\text { through a webcam to prevent } \\
\text { drowsy driving. }\end{array}$ & $\begin{array}{l}\text { Start control; } \\
\text { Accident } \\
\text { prevention }\end{array}$ & $\begin{array}{l}\text { Alcohol detection; } \\
\text { Speed detection; } \\
\text { Drowsiness } \\
\text { detection }\end{array}$ & $\begin{array}{l}\text { Single board } \\
\text { MCU } \\
\text { (Arduino) }\end{array}$ & \\
\hline $\begin{array}{l}\text { Swathi et al. } \\
\text { [46] }\end{array}$ & 2019 & $\begin{array}{l}\text { Start-up management by detecting } \\
\text { alcohol consumption and } \\
\text { helmet-wearing status, and } \\
\text { ensures security with a password } \\
\text { system }\end{array}$ & Start control & $\begin{array}{l}\text { Alcohol detection; } \\
\text { Helmet wear } \\
\text { detection }\end{array}$ & $\begin{array}{l}\text { Chip MCU } \\
\text { (Atmega8) }\end{array}$ & $\mathrm{RF}$ \\
\hline $\begin{array}{l}\text { Reddy et al. } \\
{[47]}\end{array}$ & 2019 & $\begin{array}{l}\text { Detects alcohol consumption and } \\
\text { helmet-wearing status, and sends } \\
\text { a text message in case of an } \\
\text { accident }\end{array}$ & $\begin{array}{l}\text { Start control; } \\
\text { Rescue request }\end{array}$ & $\begin{array}{l}\text { Helmet wear } \\
\text { detection; Alcohol } \\
\text { detection; Accident } \\
\text { detection }\end{array}$ & $\begin{array}{l}\text { Single board } \\
\text { MCU } \\
\text { (Arduino) }\end{array}$ & ZigBee \\
\hline $\begin{array}{l}\text { Shravya et al. } \\
\text { [48] }\end{array}$ & 2019 & $\begin{array}{l}\text { Detects whether the helmet is } \\
\text { worn, starts the engine, detects } \\
\text { alcohol consumption, locks the } \\
\text { engine, and transmits the vehicle } \\
\text { number nearby via SMS text }\end{array}$ & $\begin{array}{l}\text { Start control; } \\
\text { Rescue request }\end{array}$ & $\begin{array}{l}\text { Helmet wear } \\
\text { detection; Alcohol } \\
\text { detection; Accident } \\
\text { detection }\end{array}$ & $\begin{array}{l}\text { Single board } \\
\text { MCU } \\
\text { (Arduino) }\end{array}$ & $\mathrm{RF}$ \\
\hline
\end{tabular}


Table 2. Cont.

\begin{tabular}{|c|c|c|c|c|c|c|}
\hline Reference & Year & $\begin{array}{c}\text { Feature and High-Level } \\
\text { Functionality }\end{array}$ & $\begin{array}{c}\text { Application } \\
\text { (Sub-Category) }\end{array}$ & Sensor & Microcontroller & $\begin{array}{l}\text { Wireless } \\
\text { Technology }\end{array}$ \\
\hline $\begin{array}{l}\text { Kanimozhi et al. } \\
\text { [49] }\end{array}$ & 2019 & $\begin{array}{l}\text { Start-up management by detecting } \\
\text { whether a helmet is worn, alcohol } \\
\text { is consumed, and heart rate is } \\
\text { normal }\end{array}$ & Start control & $\begin{array}{l}\text { Helmet wear } \\
\text { detection; Alcohol } \\
\text { detection; Heart } \\
\text { rate detection }\end{array}$ & $\begin{array}{l}\text { Single board } \\
\text { MCU } \\
\text { (Arduino) }\end{array}$ & $\mathrm{RF}$ \\
\hline Patil et al. [50] & 2019 & $\begin{array}{l}\text { Detects whether the helmet is } \\
\text { worn, locks it, and detects an } \\
\text { accident by detecting a fall; sends } \\
\text { this information via text to the } \\
\text { registered number }\end{array}$ & $\begin{array}{l}\text { Start control; } \\
\text { Rescue request }\end{array}$ & $\begin{array}{l}\text { Helmet wear } \\
\text { detection; Accident } \\
\text { detection }\end{array}$ & $\begin{array}{l}\text { Chip MCU } \\
\text { (Atmega88p) }\end{array}$ & $\mathrm{RF}$ \\
\hline $\begin{array}{l}\text { Jesudoss et al. } \\
\text { [51] }\end{array}$ & 2019 & $\begin{array}{l}\text { Detects whether a helmet is worn } \\
\text { and alcohol is consumed, and } \\
\text { starts the engine when both } \\
\text { conditions are met. }\end{array}$ & Start control & $\begin{array}{l}\text { Helmet wear } \\
\text { detection; Alcohol } \\
\text { detection }\end{array}$ & $\begin{array}{l}\text { Chip MCU } \\
\text { (PIC) }\end{array}$ & ZigBee \\
\hline $\begin{array}{l}\text { Kabilan et al. } \\
\text { [52] }\end{array}$ & 2019 & $\begin{array}{l}\text { Vibration sensor detects an } \\
\text { accident and sends a text message } \\
\text { to the location of the accident }\end{array}$ & Rescue request & Accident detection & $\begin{array}{l}\text { Single board } \\
\text { computer } \\
\text { (Raspberry Pi) }\end{array}$ & WiFi \\
\hline $\begin{array}{l}\text { Ashwin and } \\
\text { Yashwanth } \\
\text { Gowda [53] }\end{array}$ & 2019 & $\begin{array}{l}\text { Detects whether a helmet is worn } \\
\text { and drinking alcohol, and when } \\
\text { both conditions are met, the } \\
\text { engine is started and a text } \\
\text { message is sent in case of an } \\
\text { accident }\end{array}$ & $\begin{array}{l}\text { Start control; } \\
\text { Rescue request }\end{array}$ & $\begin{array}{l}\text { Helmet wear } \\
\text { detection; Alcohol } \\
\text { detection; Accident } \\
\text { detection }\end{array}$ & $\begin{array}{l}\text { Chip MCU } \\
\text { (Atmega32) }\end{array}$ & $\mathrm{RF}$ \\
\hline Chen et al. [54] & 2019 & $\begin{array}{l}\text { Collision detection by analyzing } \\
\text { driver's head motion }\end{array}$ & Rescue request & $\begin{array}{l}\text { Head motion } \\
\text { detection }\end{array}$ & $\begin{array}{l}\text { Smart device } \\
\text { (Smartphone) }\end{array}$ & Bluetooth \\
\hline Gupta et al. [55] & 2019 & $\begin{array}{l}\text { Detects collision and drinking, } \\
\text { requests rescue through text } \\
\text { messages in case of an accident, } \\
\text { and acts as a black box through a } \\
\text { camera module }\end{array}$ & $\begin{array}{l}\text { Start control; } \\
\text { Rescue request }\end{array}$ & $\begin{array}{l}\text { Alcohol detection; } \\
\text { Accident detection }\end{array}$ & $\begin{array}{l}\text { Single board } \\
\text { computer } \\
\text { (Raspberry Pi) }\end{array}$ & Bluetooth \\
\hline Priya et al. [56] & 2019 & $\begin{array}{l}\text { Detects whether a helmet is worn, } \\
\text { manages the boot, detects an } \\
\text { accident with vibration, and sends } \\
\text { a text message }\end{array}$ & $\begin{array}{l}\text { Start control; } \\
\text { Rescue request }\end{array}$ & $\begin{array}{l}\text { Helmet wear } \\
\text { detection; Accident } \\
\text { detection }\end{array}$ & $\begin{array}{l}\text { Chip MCU } \\
\text { (PIC) }\end{array}$ & $\mathrm{RF}$ \\
\hline $\begin{array}{l}\text { Vijayakumar } \\
\text { et al. [57] }\end{array}$ & 2019 & $\begin{array}{l}\text { Detects whether a helmet is worn } \\
\text { and drinking, and notifies the } \\
\text { driver of the presence of nearby } \\
\text { vehicles through motion detection }\end{array}$ & $\begin{array}{l}\text { Start control; } \\
\text { Accident } \\
\text { prevention }\end{array}$ & $\begin{array}{l}\text { Helmet wear } \\
\text { detection; Alcohol } \\
\text { detection; Motion } \\
\text { detection }\end{array}$ & $\begin{array}{l}\text { Single board } \\
\text { MCU } \\
\text { (Arduino) }\end{array}$ & $\mathrm{RF}$ \\
\hline $\begin{array}{l}\text { Divyasudha } \\
\text { et al. [58] }\end{array}$ & 2019 & $\begin{array}{l}\text { Accident, location, and alcohol are } \\
\text { detected and sent to the cloud } \\
\text { server for analysis }\end{array}$ & $\begin{array}{l}\text { Start control; } \\
\text { Rescue request }\end{array}$ & $\begin{array}{l}\text { Alcohol detection; } \\
\text { Alcohol detection; } \\
\text { Location detection }\end{array}$ & $\begin{array}{l}\text { Single board } \\
\text { MCU } \\
\text { (Arduino) }\end{array}$ & ZigBee \\
\hline $\begin{array}{l}\text { Mhatre et al. } \\
\text { [59] }\end{array}$ & 2020 & $\begin{array}{l}\text { Detects whether a helmet is worn } \\
\text { and drinking alcohol, and when } \\
\text { both conditions are met, the } \\
\text { engine starts and a text message is } \\
\text { sent to the designated number in } \\
\text { case of an accident }\end{array}$ & $\begin{array}{l}\text { Start control; } \\
\text { Rescue request }\end{array}$ & $\begin{array}{l}\text { Helmet wear } \\
\text { detection; Alcohol } \\
\text { detection; Accident } \\
\text { detection }\end{array}$ & $\begin{array}{l}\text { Single board } \\
\text { MCU } \\
\text { (Arduino) }\end{array}$ & $\mathrm{RF}$ \\
\hline $\begin{array}{l}\text { Lakshmanachari } \\
\text { et al. [60] }\end{array}$ & 2020 & $\begin{array}{l}\text { Detects whether a helmet is worn, } \\
\text { drinking, or speeding, and sends a } \\
\text { text message in case of an accident }\end{array}$ & $\begin{array}{l}\text { Start control; } \\
\text { Rescue request }\end{array}$ & $\begin{array}{l}\text { Helmet wear } \\
\text { detection; Alcohol } \\
\text { detection; Speed } \\
\text { detection; Accident } \\
\text { detection }\end{array}$ & $\begin{array}{l}\text { Chip MCU } \\
\text { (ARM7) }\end{array}$ & $\mathrm{RF}$ \\
\hline $\begin{array}{l}\text { Merlin and } \\
\text { Pranay [61] }\end{array}$ & 2020 & $\begin{array}{l}\text { Detects whether the helmet is } \\
\text { worn and sounds the buzzer until } \\
\text { the helmet is worn }\end{array}$ & Start control & $\begin{array}{l}\text { Helmet wear } \\
\text { detection }\end{array}$ & $\begin{array}{l}\text { Single board } \\
\text { MCU } \\
\text { (Arduino) }\end{array}$ & $\mathrm{RF}$ \\
\hline $\begin{array}{l}\text { Chidambarathanu } \\
\text { et al. [62] }\end{array}$ & 2020 & $\begin{array}{l}\text { Collects brainwave and drinking } \\
\text { data and sends location } \\
\text { information via text in case of an } \\
\text { accident }\end{array}$ & $\begin{array}{l}\text { Start control; } \\
\text { Rescue request }\end{array}$ & $\begin{array}{l}\text { Brainwave } \\
\text { detection; Alcohol } \\
\text { detection; Accident } \\
\text { detection }\end{array}$ & $\begin{array}{l}\text { Single board } \\
\text { MCU } \\
\text { (Arduino) }\end{array}$ & Bluetooth \\
\hline
\end{tabular}


Table 2. Cont.

\begin{tabular}{|c|c|c|c|c|c|c|}
\hline Reference & Year & $\begin{array}{l}\text { Feature and High-Level } \\
\text { Functionality }\end{array}$ & $\begin{array}{c}\text { Application } \\
\text { (Sub-Category) }\end{array}$ & Sensor & Microcontroller & $\begin{array}{l}\text { Wireless } \\
\text { Technology }\end{array}$ \\
\hline $\begin{array}{l}\text { Santhanakrishnan } \\
\text { et al. [63] }\end{array}$ & 2020 & $\begin{array}{l}\text { Detects whether or not to wear a } \\
\text { helmet and drinking alcohol, and } \\
\text { when both conditions are met, the } \\
\text { engine is started and location } \\
\text { information is sent by text in case } \\
\text { of an accident }\end{array}$ & $\begin{array}{l}\text { Start control; } \\
\text { Rescue request }\end{array}$ & $\begin{array}{l}\text { Helmet wear } \\
\text { detection; Alcohol } \\
\text { detection; Accident } \\
\text { detection; Location } \\
\text { detection }\end{array}$ & $\begin{array}{l}\text { Single board } \\
\text { MCU } \\
\text { (Arduino) }\end{array}$ & RF \\
\hline $\begin{array}{l}\text { Sai Kumar and } \\
\text { Aruna [64] }\end{array}$ & 2020 & $\begin{array}{l}\text { Detects whether a helmet is worn, } \\
\text { and when not wearing a message, } \\
\text { a message is displayed through } \\
\text { the LCD, and a text message is } \\
\text { sent to detect alcohol and } \\
\text { accidents }\end{array}$ & $\begin{array}{l}\text { Start control; } \\
\text { Rescue request }\end{array}$ & $\begin{array}{l}\text { Helmet wear } \\
\text { detection; Alcohol } \\
\text { detection; Accident } \\
\text { detection }\end{array}$ & $\begin{array}{l}\text { Chip MCU } \\
\text { (PIC) }\end{array}$ & ZigBee \\
\hline $\begin{array}{l}\text { Suman et al. } \\
{[65]}\end{array}$ & 2020 & $\begin{array}{l}\text { Detects drinking alcohol and } \\
\text { wearing a helmet and sends a text } \\
\text { message in case of an accident }\end{array}$ & $\begin{array}{l}\text { Start control; } \\
\text { Rescue request }\end{array}$ & $\begin{array}{l}\text { Alcohol detection; } \\
\text { Helmet wear } \\
\text { detection; Accident } \\
\text { detection }\end{array}$ & $\begin{array}{l}\text { Chip MCU } \\
\text { (TICC2200) }\end{array}$ & WiFi \\
\hline $\begin{array}{l}\text { Ahmed et al. } \\
\text { [66] }\end{array}$ & 2020 & $\begin{array}{l}\text { Detects objects, detects danger, } \\
\text { and sends text messages in case of } \\
\text { an accident }\end{array}$ & Rescue request & $\begin{array}{l}\text { Object detection; } \\
\text { accident detection }\end{array}$ & $\begin{array}{l}\text { Single board } \\
\text { computer } \\
\text { (Raspberry Pi) }\end{array}$ & WiFi \\
\hline $\begin{array}{l}\text { Dubey et al. } \\
\text { [67] }\end{array}$ & 2020 & $\begin{array}{l}\text { Detects whether a helmet is worn; } \\
\text { monitors road conditions to help } \\
\text { change lanes; and sends a text } \\
\text { message in case of an accident }\end{array}$ & $\begin{array}{l}\text { Start control; } \\
\text { Rescue request }\end{array}$ & $\begin{array}{l}\text { Helmet wear } \\
\text { detection; Accident } \\
\text { detection }\end{array}$ & $\begin{array}{l}\text { Single board } \\
\text { MCU } \\
\text { (Arduino) }\end{array}$ & \\
\hline $\begin{array}{l}\text { Lokeshwaran } \\
\text { et al. [68] }\end{array}$ & 2020 & $\begin{array}{l}\text { Detect wearing a helmet, measures } \\
\text { your heart rate, and makes a call } \\
\text { in case of an accident }\end{array}$ & $\begin{array}{l}\text { Start control; } \\
\text { Rescue request }\end{array}$ & $\begin{array}{l}\text { Helmet wear } \\
\text { detection; Accident } \\
\text { detection; Heart } \\
\text { rate detection }\end{array}$ & $\begin{array}{l}\text { Single board } \\
\text { MCU } \\
\text { (NODEMCU) }\end{array}$ & RF \\
\hline $\begin{array}{l}\text { Rahman et al. } \\
\text { [69] }\end{array}$ & 2020 & $\begin{array}{l}\text { Detects drinking and wearing a } \\
\text { helmet, stores location } \\
\text { information, and transmits } \\
\text { location information in case of an } \\
\text { accident }\end{array}$ & $\begin{array}{l}\text { Start control; } \\
\text { Rescue request }\end{array}$ & $\begin{array}{l}\text { Helmet wear } \\
\text { detection; Alcohol } \\
\text { detection; Accident } \\
\text { detection; Location } \\
\text { detection }\end{array}$ & $\begin{array}{l}\text { Single board } \\
\text { MCU } \\
\text { (Arduino) }\end{array}$ & Bluetooth \\
\hline Faikul et al. [70] & 2020 & $\begin{array}{l}\text { Prevents drowsy driving by } \\
\text { detecting heart rate }\end{array}$ & $\begin{array}{l}\text { Accident } \\
\text { prevention }\end{array}$ & Heart rate detection & $\begin{array}{l}\text { Single board } \\
\text { MCU } \\
\text { (Arduino) }\end{array}$ & \\
\hline Rao et al. [71] & 2020 & $\begin{array}{l}\text { Detects wearing of a helmet and } \\
\text { drinking, manages the start-up, } \\
\text { and sends location information via } \\
\text { text message }\end{array}$ & $\begin{array}{l}\text { Start control; } \\
\text { Rescue request }\end{array}$ & $\begin{array}{l}\text { Helmet wear } \\
\text { detection; Alcohol } \\
\text { detection; Accident } \\
\text { detection; Location } \\
\text { detection }\end{array}$ & $\begin{array}{l}\text { Single board } \\
\text { MCU } \\
\text { (Arduino) }\end{array}$ & WiFi \\
\hline $\begin{array}{l}\text { Oviyaa et al. } \\
\text { [72] }\end{array}$ & 2020 & $\begin{array}{l}\text { Brainwave is measured to detect } \\
\text { drowsiness and fatigue and give a } \\
\text { warning }\end{array}$ & $\begin{array}{l}\text { Accident } \\
\text { prevention }\end{array}$ & $\begin{array}{l}\text { Brainwave } \\
\text { detection }\end{array}$ & $\begin{array}{l}\text { Single board } \\
\text { MCU } \\
\text { (Arduino) }\end{array}$ & Bluetooth \\
\hline $\begin{array}{l}\text { Shahare et al. } \\
\text { [73] }\end{array}$ & 2020 & $\begin{array}{l}\text { Detects wearing a helmet, } \\
\text { manages the start-up, detects } \\
\text { alcohol consumption and } \\
\text { accidents, and sends location } \\
\text { information by text in case of an } \\
\text { accident }\end{array}$ & $\begin{array}{l}\text { Start control; } \\
\text { Rescue request }\end{array}$ & $\begin{array}{l}\text { Helmet wear } \\
\text { detection, Alcohol } \\
\text { detection; Accident } \\
\text { detection; Location } \\
\text { detection }\end{array}$ & $\begin{array}{l}\text { Single board } \\
\text { MCU } \\
\text { (Arduino) }\end{array}$ & RF \\
\hline $\begin{array}{l}\text { Ashwini et al. } \\
\text { [74] }\end{array}$ & 2020 & $\begin{array}{l}\text { Detects wearing a helmet, } \\
\text { manages the start-up, detects } \\
\text { drinking and accidents, and sends } \\
\text { location information by text in } \\
\text { case of an accident }\end{array}$ & $\begin{array}{l}\text { Start control; } \\
\text { Rescue request }\end{array}$ & $\begin{array}{l}\text { Helmet wear } \\
\text { detection; Alcohol } \\
\text { detection; Accident } \\
\text { detection; Location } \\
\text { detection }\end{array}$ & $\begin{array}{l}\text { Single board } \\
\text { MCU } \\
\text { (Arduino) }\end{array}$ & $\mathrm{RF}$ \\
\hline $\begin{array}{l}\text { Jayasinghe and } \\
\text { Arachchige [75] }\end{array}$ & 2020 & $\begin{array}{l}\text { Detects alcohol and drowsiness } \\
\text { and manages start-up }\end{array}$ & $\begin{array}{l}\text { Start control; } \\
\text { Accident } \\
\text { prevention; } \\
\text { Rescue request }\end{array}$ & $\begin{array}{l}\text { Alcohol detection; } \\
\text { Drowsiness } \\
\text { detection }\end{array}$ & $\begin{array}{l}\text { Single board } \\
\text { MCU } \\
\text { (Arduino) }\end{array}$ & Bluetooth \\
\hline
\end{tabular}


Table 2. Cont.

\begin{tabular}{|c|c|c|c|c|c|c|}
\hline Reference & Year & $\begin{array}{l}\text { Feature and High-Level } \\
\text { Functionality }\end{array}$ & $\begin{array}{c}\text { Application } \\
\text { (Sub-Category) }\end{array}$ & Sensor & Microcontroller & $\begin{array}{l}\text { Wireless } \\
\text { Technology }\end{array}$ \\
\hline $\begin{array}{l}\text { Aravinda et al. } \\
\text { [76] }\end{array}$ & 2020 & $\begin{array}{l}\text { Detects wearing a helmet and } \\
\text { manages the start-up }\end{array}$ & Start control & $\begin{array}{l}\text { Helmet wear } \\
\text { detection }\end{array}$ & $\begin{array}{l}\text { Single board } \\
\text { computer } \\
\text { (Raspberry Pi) }\end{array}$ & $\mathrm{RF}$ \\
\hline $\begin{array}{l}\text { MohanaRoopa } \\
\text { et al. [77] }\end{array}$ & 2020 & $\begin{array}{l}\text { Start management by detecting } \\
\text { drinking, wearing a helmet, and } \\
\text { sending location information by } \\
\text { text in case of an accident }\end{array}$ & $\begin{array}{l}\text { Start control; } \\
\text { Rescue request }\end{array}$ & $\begin{array}{l}\text { Helmet wear } \\
\text { detection; Alcohol } \\
\text { detection; Accident } \\
\text { detection; Location } \\
\text { detection }\end{array}$ & $\begin{array}{l}\text { Single board } \\
\text { MCU } \\
\text { (Arduino) }\end{array}$ & WiFi \\
\hline $\begin{array}{l}\text { Pothirajan and } \\
\text { Mary [78] }\end{array}$ & 2020 & $\begin{array}{l}\text { Detects drinking and wearing a } \\
\text { helmet }\end{array}$ & Start control & $\begin{array}{l}\text { Helmet wear } \\
\text { detection; Alcohol } \\
\text { detection }\end{array}$ & $\begin{array}{l}\text { Single board } \\
\text { MCU } \\
\text { (Arduino) }\end{array}$ & $\mathrm{RF}$ \\
\hline $\begin{array}{l}\text { Swarna et al. } \\
\text { [79] }\end{array}$ & 2020 & $\begin{array}{l}\text { Detects alcohol and manages the } \\
\text { start-up and sends location } \\
\text { information via text message in } \\
\text { case of an accident }\end{array}$ & $\begin{array}{l}\text { Start control; } \\
\text { Rescue request }\end{array}$ & $\begin{array}{l}\text { Alcohol detection; } \\
\text { Location detection }\end{array}$ & $\begin{array}{l}\text { Single board } \\
\text { MCU } \\
\text { (Arduino) }\end{array}$ & $\mathrm{RF}$ \\
\hline $\begin{array}{l}\text { Parakkal et al. } \\
\text { [80] }\end{array}$ & 2020 & $\begin{array}{l}\text { Detects speeding and sends } \\
\text { location information via text in } \\
\text { case of an accident }\end{array}$ & $\begin{array}{l}\text { Start control; } \\
\text { Accident } \\
\text { prevention; } \\
\text { Rescue request }\end{array}$ & $\begin{array}{l}\text { Speed detection; } \\
\text { Location detection }\end{array}$ & $\begin{array}{l}\text { Single board } \\
\text { computer } \\
\text { (Raspberry Pi) }\end{array}$ & WiFi \\
\hline $\begin{array}{l}\text { Kumar Kar } \\
\text { et al. [81] }\end{array}$ & 2018 & $\begin{array}{l}\text { Convenience is provided through } \\
\text { systems such as headlights and } \\
\text { audio }\end{array}$ & $\begin{array}{l}\text { Convenience } \\
\text { improvement }\end{array}$ & Location detection & $\begin{array}{l}\text { Single board } \\
\text { MCU } \\
\text { (Arduino) }\end{array}$ & Bluetooth \\
\hline $\begin{array}{l}\text { Rahman et al. } \\
\text { [82] }\end{array}$ & 2019 & $\begin{array}{l}\text { Detect fuel level and notify } \\
\text { through notification system }\end{array}$ & $\begin{array}{l}\text { Convenience } \\
\text { improvement }\end{array}$ & Fuel level detection & $\begin{array}{l}\text { Single board } \\
\text { MCU } \\
\text { (Arduino) }\end{array}$ & Bluetooth \\
\hline $\begin{array}{l}\text { Jadhav et al. } \\
\text { [83] }\end{array}$ & 2019 & $\begin{array}{l}\text { Provides convenience, such as } \\
\text { navigation, traffic conditions, } \\
\text { music, and phone calls, through } \\
\text { voice recognition on the HUD by } \\
\text { connecting a mobile phone }\end{array}$ & $\begin{array}{l}\text { Convenience } \\
\text { improvement }\end{array}$ & Location detection & $\begin{array}{l}\text { Single board } \\
\text { MCU } \\
\text { (Arduino) }\end{array}$ & Bluetooth \\
\hline $\begin{array}{l}\text { Youssef et al. } \\
\text { [84] }\end{array}$ & 2019 & $\begin{array}{l}\text { Provides thermal comfort when } \\
\text { wearing a helmet }\end{array}$ & $\begin{array}{l}\text { Convenience } \\
\text { improvement }\end{array}$ & $\begin{array}{l}\text { Humidity detection; } \\
\text { Temperature } \\
\text { detection }\end{array}$ & $\begin{array}{l}\text { Single board } \\
\text { MCU } \\
\text { (Arduino) }\end{array}$ & Bluetooth \\
\hline Rao [85] & 2019 & $\begin{array}{l}\text { A system that can operate the } \\
\text { helmet wiper through voice } \\
\text { recognition }\end{array}$ & $\begin{array}{l}\text { Convenience } \\
\text { improvement }\end{array}$ & $\mathrm{N} / \mathrm{A}$ & $\begin{array}{l}\text { Single board } \\
\text { MCU } \\
\text { (Arduino) }\end{array}$ & Wifi \\
\hline $\begin{array}{l}\text { Kanetkar et al. } \\
{[86]}\end{array}$ & 2020 & $\begin{array}{l}\text { Detects raindrops and } \\
\text { automatically controls the } \\
\text { helmet's wiper }\end{array}$ & $\begin{array}{l}\text { Convenience } \\
\text { improvement }\end{array}$ & Rain detection & $\begin{array}{l}\text { Single board } \\
\text { MCU } \\
\text { (Arduino) }\end{array}$ & $\mathrm{N} / \mathrm{A}$ \\
\hline $\begin{array}{l}\text { Astif and } \\
\text { Manoj [87] }\end{array}$ & 2017 & $\begin{array}{l}\text { Convenience such as navigation } \\
\text { and listening to music is provided, } \\
\text { and a text message is sent by } \\
\text { pressing a button in case of an } \\
\text { accident }\end{array}$ & $\begin{array}{l}\text { Rescue request; } \\
\text { Convenience } \\
\text { improvement }\end{array}$ & Location detection & $\begin{array}{l}\text { Single board } \\
\text { MCU } \\
\text { (Arduino) }\end{array}$ & Bluetooth \\
\hline Ajay et al. [88] & 2017 & $\begin{array}{l}\text { Provides convenience functions } \\
\text { while driving and voice calls in } \\
\text { case of an accident }\end{array}$ & $\begin{array}{l}\text { Rescue request; } \\
\text { Convenience } \\
\text { improvement }\end{array}$ & Accident detection & $\begin{array}{l}\text { Single board } \\
\text { MCU } \\
\text { (Arduino) }\end{array}$ & Bluetooth \\
\hline $\begin{array}{l}\text { Kumar et al. } \\
{[89]}\end{array}$ & 2019 & $\begin{array}{l}\text { By connecting to a mobile phone, } \\
\text { it provides convenience, such as } \\
\text { phone calls, navigation, and } \\
\text { listening to music, and detects } \\
\text { alcohol consumption }\end{array}$ & $\begin{array}{l}\text { Rescue request; } \\
\text { Convenience } \\
\text { improvement }\end{array}$ & Alcohol detection & $\begin{array}{l}\text { Single board } \\
\text { MCU } \\
\text { (Arduino) }\end{array}$ & Bluetooth \\
\hline
\end{tabular}

For example, in this category, Kanimozhi et al. [49] developed a smart helmet using a single board-based microcontroller (Arduino) with several sensors for detecting alcohol gas (MQ3) and heart pulse of motorcyclists. The radio frequency (RF) module was used to transmit and/or receive radio signals between the smart helmet and motorcycle. The data were stored in a local storage medium because the helmet did not provide a function 
to share the data that it monitored with other users. Uniyal et al. [31] developed a smart helmet using the Arduino board, RF transmitter, and sensors for detecting helmet wear, speed, accident, and location. The smart helmet collects and records speeding data of the bike and provides a risk warning when the bike exceeds the speed limit.

A total of 22 academic papers that deal with the applications of smart helmets for workers were published during the past 11 years [8,9,90-109] and are summarized in Table 3. The application fields include mining, construction, petrochemistry, disaster prevention, and medical treatment. Figure 6 shows the percentage of smart helmet studies conducted in each application field.

Table 3. Summary of smart helmet applications for workers in the fields of mining, construction, petrochemistry, disaster prevention, medical treatment, etc.

\begin{tabular}{|c|c|c|c|c|c|c|}
\hline Reference & Year & $\begin{array}{c}\text { Feature and High-Level } \\
\text { Functionality }\end{array}$ & $\begin{array}{c}\text { Application } \\
\text { (Sub-Category) }\end{array}$ & Sensor & Microcontroller & $\begin{array}{l}\text { Wireless } \\
\text { Technology }\end{array}$ \\
\hline Wang et al. [8] & 2020 & $\begin{array}{l}\text { Detects stair fall by analyzing } \\
\text { changes in weight support and } \\
\text { pressure center }\end{array}$ & Construction & Accident detection & $\begin{array}{l}\text { Single board } \\
\text { MCU } \\
\text { (Arduino) }\end{array}$ & Bluetooth \\
\hline $\begin{array}{l}\text { Mohammed } \\
\text { et al. [9] }\end{array}$ & 2020 & $\begin{array}{l}\text { Helps prevent the spread of } \\
\text { coronavirus by measuring the } \\
\text { body temperature and personal } \\
\text { information of pedestrians }\end{array}$ & $\begin{array}{l}\text { Medical } \\
\text { treatment }\end{array}$ & $\begin{array}{l}\text { Temperature } \\
\text { detection }\end{array}$ & $\begin{array}{l}\text { Single board } \\
\text { MCU } \\
\text { (Arduino) }\end{array}$ & Bluetooth \\
\hline Qiang et al. [90] & 2009 & $\begin{array}{l}\text { Real-time monitoring of methane, } \\
\text { temperature and humidity data } \\
\text { and support for voice } \\
\text { communication between operators }\end{array}$ & Mining & $\begin{array}{l}\text { Harmful gas } \\
\text { detection; Humidity } \\
\text { detection; } \\
\text { Temperature } \\
\text { detection }\end{array}$ & $\begin{array}{l}\text { Chip MCU } \\
\text { (S3C44BOX) }\end{array}$ & ZigBee \\
\hline Shabina [91] & 2014 & $\begin{array}{l}\text { Transmits real-time temperature, } \\
\text { humidity, and fire data through a } \\
\text { wireless network and alerts the } \\
\text { operator by sounding an alarm } \\
\text { when the threshold is reached }\end{array}$ & Mining & $\begin{array}{l}\text { Temperature } \\
\text { detection; Humidity } \\
\text { detection; Fire } \\
\text { detection }\end{array}$ & $\begin{array}{l}\text { Chip MCU } \\
\text { (AT89S52) }\end{array}$ & N/A \\
\hline Behr et al. [92] & 2016 & $\begin{array}{l}\text { Collects data on the concentration } \\
\text { of harmful gases, whether the } \\
\text { helmet is worn, and the amount of } \\
\text { impact on the helmet, and } \\
\text { transmits and analyzes it to the } \\
\text { cloud server }\end{array}$ & Mining & $\begin{array}{l}\text { Harmful gas } \\
\text { detection; Helmet } \\
\text { wear detection }\end{array}$ & $\begin{array}{l}\text { Chip MCU } \\
\text { (ATZB-24-A2) }\end{array}$ & ZigBee \\
\hline Hazarika [93] & 2017 & $\begin{array}{l}\text { Measures the concentration of } \\
\text { harmful gas and sends it to the } \\
\text { cloud server for real-time } \\
\text { monitoring and alarms in the } \\
\text { control room when the threshold } \\
\text { is exceeded }\end{array}$ & Mining & $\begin{array}{l}\text { Harmful gas } \\
\text { detection }\end{array}$ & $\begin{array}{l}\text { Single board } \\
\text { MCU } \\
\text { (Arduino) }\end{array}$ & ZigBee \\
\hline $\begin{array}{l}\text { Sharma and } \\
\text { Maity [94] }\end{array}$ & 2018 & $\begin{array}{l}\text { Measures the concentration of } \\
\text { harmful gases, temperature, and } \\
\text { humidity, sends them to the cloud } \\
\text { server for real-time monitoring, } \\
\text { and sounds an alarm when the } \\
\text { threshold is exceeded }\end{array}$ & Mining & $\begin{array}{l}\text { Harmful gas } \\
\text { detection; } \\
\text { Temperature } \\
\text { detection; Humidity } \\
\text { detection }\end{array}$ & $\begin{array}{l}\text { Single board } \\
\text { MCU } \\
\text { (Arduino) }\end{array}$ & ZigBee \\
\hline $\begin{array}{l}\text { Revindran et al. } \\
\text { [95] }\end{array}$ & 2018 & $\begin{array}{l}\text { A wireless sensor network was } \\
\text { built and rescue request was from } \\
\text { nearby workers and medical } \\
\text { teams when workers send distress } \\
\text { messages }\end{array}$ & Mining & Accident detection & $\begin{array}{l}\text { Chip MCU } \\
\text { (Arduino) }\end{array}$ & $\mathrm{RF}$ \\
\hline $\begin{array}{l}\text { Eldemerdash } \\
\text { et al. [96] }\end{array}$ & 2019 & $\begin{array}{l}\text { Temperature and humidity, } \\
\text { harmful gas concentration, and } \\
\text { pressure data are monitored, and } \\
\text { when thresholds are reached, } \\
\text { warnings are sent through LEDs } \\
\text { and buzzers }\end{array}$ & Mining & $\begin{array}{l}\text { Temperature } \\
\text { detection; Humidity } \\
\text { detection; Harmful } \\
\text { gas detection }\end{array}$ & $\begin{array}{l}\text { Single board } \\
\text { computer } \\
\text { (Raspberry Pi) }\end{array}$ & ZigBee \\
\hline
\end{tabular}


Table 3. Cont.

\begin{tabular}{|c|c|c|c|c|c|c|}
\hline Reference & Year & $\begin{array}{l}\text { Feature and High-Level } \\
\text { Functionality }\end{array}$ & $\begin{array}{c}\text { Application } \\
\text { (Sub-Category) }\end{array}$ & Sensor & Microcontroller & $\begin{array}{l}\text { Wireless } \\
\text { Technology }\end{array}$ \\
\hline $\begin{array}{l}\text { Sanjay et al. } \\
\text { [97] }\end{array}$ & 2019 & $\begin{array}{l}\text { Temperature and humidity, } \\
\text { harmful gas concentration, and } \\
\text { pressure data are monitored, and } \\
\text { when thresholds are reached, } \\
\text { warnings are made through LEDs } \\
\text { and buzzers }\end{array}$ & Mining & $\begin{array}{l}\text { Temperature } \\
\text { detection; Humidity } \\
\text { detection; Harmful } \\
\text { gas detection }\end{array}$ & $\begin{array}{l}\text { Single board } \\
\text { MCU } \\
\text { (Arduino) }\end{array}$ & ZigBee \\
\hline $\begin{array}{l}\text { Charde et al. } \\
\text { [98] }\end{array}$ & 2020 & $\begin{array}{l}\text { Collects harmful gas } \\
\text { concentration, humidity, and } \\
\text { temperature data to enable } \\
\text { real-time monitoring and alerts } \\
\text { through buzzer and LCD when } \\
\text { hazardous concentrations are } \\
\text { reached }\end{array}$ & Mining & $\begin{array}{l}\text { Temperature } \\
\text { detection; Humidity } \\
\text { detection; Harmful } \\
\text { gas detection }\end{array}$ & $\begin{array}{l}\text { Chip MCU } \\
\text { (PIC) }\end{array}$ & ZigBee \\
\hline $\begin{array}{l}\text { Sujitha et al. } \\
\text { [99] }\end{array}$ & 2020 & $\begin{array}{l}\text { Detects temperature and humidity, } \\
\text { light intensity, toxic gas levels in } \\
\text { the air, traces of flames, etc. and } \\
\text { warns when thresholds are } \\
\text { exceeded }\end{array}$ & Mining & $\begin{array}{l}\text { Temperature and } \\
\text { Humidity detection; } \\
\text { Brightness } \\
\text { detection; Harmful } \\
\text { gas detection; Fire } \\
\text { detection }\end{array}$ & $\begin{array}{l}\text { Single board } \\
\text { MCU } \\
\text { (Arduino) }\end{array}$ & WiFi \\
\hline Pirkl et al. [100] & 2016 & $\begin{array}{l}\text { IR camera sensor measures the } \\
\text { temperature of the surrounding } \\
\text { environment }\end{array}$ & Construction & $\begin{array}{l}\text { Temperature } \\
\text { detection }\end{array}$ & $\begin{array}{l}\text { Chip MCU } \\
\text { (IntelEdison) }\end{array}$ & Bluetooth \\
\hline Lee et al. [101] & 2019 & $\begin{array}{l}\text { Detects falls, air quality, and } \\
\text { objects fall and sends data to the } \\
\text { cloud server for storage }\end{array}$ & Construction & $\begin{array}{l}\text { Harmful gas } \\
\text { detection; Accident } \\
\text { detection }\end{array}$ & $\begin{array}{l}\text { Single board } \\
\text { MCU } \\
\text { (Arduino) }\end{array}$ & Bluetooth \\
\hline Li et al. [102] & 2014 & $\begin{array}{l}\text { Inertia and brain waves are } \\
\text { measured to analyze injury and } \\
\text { fatigue, and vibration alerts when } \\
\text { thresholds are exceeded }\end{array}$ & General & $\begin{array}{l}\text { Brainwave } \\
\text { detection; Motion } \\
\text { detection }\end{array}$ & $\begin{array}{l}\text { Chip MCU } \\
\text { (PIC) }\end{array}$ & $\mathrm{N} / \mathrm{A}$ \\
\hline $\begin{array}{l}\text { Dhingra et al. } \\
\text { [103] }\end{array}$ & 2018 & $\begin{array}{l}\text { Voice communication between } \\
\text { workers is possible and rescue } \\
\text { request through panic button in } \\
\text { case of crisis }\end{array}$ & General & Accident detection & $\begin{array}{l}\text { Single board } \\
\text { MCU } \\
\text { (Arduino) }\end{array}$ & RF \\
\hline $\begin{array}{l}\text { Aston et al. } \\
\text { [104] }\end{array}$ & 2020 & Impulse quantity is dispersed & General & $\mathrm{N} / \mathrm{A}$ & Chip MCU & $\mathrm{N} / \mathrm{A}$ \\
\hline $\begin{array}{l}\text { Campero- } \\
\text { Jurado et al. } \\
{[105]}\end{array}$ & 2020 & $\begin{array}{l}\text { Temperature and humidity, } \\
\text { harmful gas concentration and } \\
\text { brightness are detected and } \\
\text { transmitted to a cloud server for } \\
\text { analysis }\end{array}$ & General & $\begin{array}{l}\text { Temperature and } \\
\text { Humidity detection; } \\
\text { Brightness } \\
\text { detection; Harmful } \\
\text { gas detection }\end{array}$ & $\begin{array}{l}\text { Chip MCU } \\
\text { (PIC) }\end{array}$ & WiFi \\
\hline Shu et al. [106] & 2015 & $\begin{array}{l}\text { Early warning in case of leakage of } \\
\text { harmful gas }\end{array}$ & Petrochemistry & $\begin{array}{l}\text { Harmful gas } \\
\text { detection }\end{array}$ & $\begin{array}{l}\text { Smart device } \\
\text { (Smartwatch) }\end{array}$ & WiFi \\
\hline Bisio et al. [107] & 2017 & $\begin{array}{l}\text { Detects stroke in emergency } \\
\text { patients }\end{array}$ & $\begin{array}{l}\text { Medical } \\
\text { treatment }\end{array}$ & $\begin{array}{l}\text { Brainwave } \\
\text { detection }\end{array}$ & $\begin{array}{l}\text { Smart device } \\
\text { (Smartphone) }\end{array}$ & Bluetooth \\
\hline $\begin{array}{l}\text { Shahiduzzaman } \\
\text { et al. [108] }\end{array}$ & 2019 & $\begin{array}{l}\text { Detects a fall, sends medical data } \\
\text { to the medical cloud, and requests } \\
\text { rescue }\end{array}$ & $\begin{array}{l}\text { Medical } \\
\text { treatment }\end{array}$ & Accident detection & $\begin{array}{l}\text { Smart device } \\
\text { (Smartphone) }\end{array}$ & Bluetooth \\
\hline $\begin{array}{l}\text { Jeong et al. } \\
\text { [109] }\end{array}$ & 2018 & $\begin{array}{l}\text { Infrared image, optical image, } \\
\text { drone image, oxygen residual } \\
\text { amount, inertia, etc. are collected }\end{array}$ & $\begin{array}{l}\text { Disaster } \\
\text { prevention }\end{array}$ & $\begin{array}{l}\text { Harmful gas } \\
\text { detection }\end{array}$ & $\begin{array}{l}\text { Smart device } \\
\text { (Smartwatch) }\end{array}$ & WiFi \\
\hline
\end{tabular}




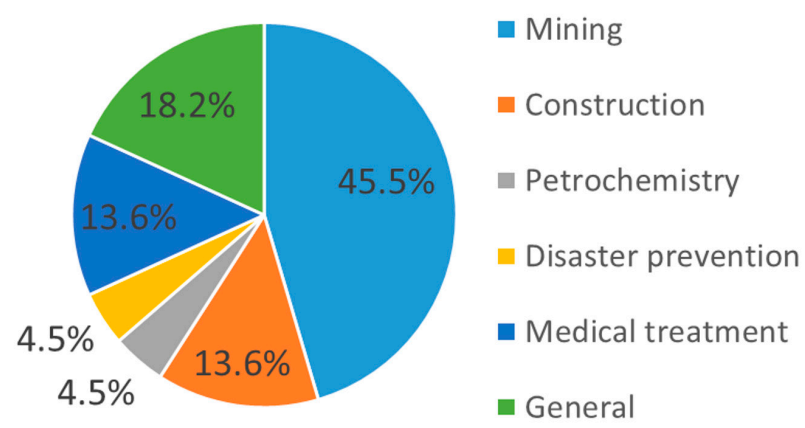

Figure 6. Percentage of smart helmet studies for workers by sub-category.

In the mining industry, 10 studies were performed on a smart helmet that detects harmful gases, such as carbon monoxide $(\mathrm{CO})$ and methane $\left(\mathrm{CH}_{4}\right)$, and guarantees the safety of workers through rescue requests in the case of a dangerous situation [90-99]. Three studies have been reported in the construction industry, where smart helmets were primarily used to detect workplace hazards and provide risk alarms to construction workers $[8,100,101]$. For example, a safety helmet with gravity and head movement sensors was used at the construction site to detect a falling worker and activate emergency rescue requests [8]. Four studies on smart helmets were conducted to support voice communication between workers and warn them by detecting injuries in general manufacturing sites [102-105]. A smart helmet that ensures the safety of workers by warning the occurrence of harmful gas leaks was developed in the petrochemical industry [106].

Three studies $[9,107,108]$ on smart helmets were found in the medical field to improve the work efficiency of medical staff and ensure the safety of patients. In this application, a smart helmet worn by the medical staff was used to detect body temperature and brain waves in emergency situations $[9,107]$. A smart helmet was developed for medical staff to detect the body temperature of a pedestrian in real time. It was used to prevent coronavirus by warning them through an alarm when discovering a high-temperature pedestrian. Additionally, a study developed a smart helmet to prevent elderly patients lying in bed from falling [108]. One study reported in the literature dealt with a smart helmet for disaster prevention [109]. In this study, the smart helmet was used to support rescue workers at disaster sites.

\subsection{Sensors Used for Smart Helmet Applications}

Various sensors are attached to a smart helmet based on the application field and purpose. Table 4 shows the types of sensors used in smart helmet studies. The sensors can be broadly classified into three types: human health, environment, and machine monitoring. The cumulative number of sensor types used in smart helmet studies by year is illustrated in Figure 7a. The use of sensors to monitor human health is increasing every year. The frequency of use of environmental monitoring sensors has also increased in recent years. The percentages of sensor uses for each sensor type are shown in Figure 7b-d. For human health monitoring, the accident detection sensor accounts for $32 \%$, followed by the helmet wear detection sensor and alcohol detection sensor with $30 \%$ and $27 \%$, respectively. In the case of environmental monitoring, sensors for harmful gas detection, video shooting, and temperature detection each account for $23 \%$. For the machine monitoring, the speed detection sensor occupies $70 \%$ and is the most used. 
Table 4. Types of sensor depending on the purpose used in smart helmet studies.

\begin{tabular}{lll}
\hline \multicolumn{1}{c}{ Human Health Monitoring } & \multicolumn{1}{c}{ Environmental Monitoring } & \multicolumn{1}{c}{ Machine Monitoring } \\
\hline - Accident detection (50 papers) & - Harmful gas detection (11 papers) & - Speed detection (7 papers) \\
- Helmet wear detection & - Video shooting (11 papers) & - Proximity warning (2 papers) \\
(46 paper) & - Temperature detection (11 papers) & - Fuel level detection (1 papers) \\
- Alcohol detection (42 papers) & - Humidity detection (7 papers) & \\
- Drowsiness detection & - Brightness detection (3 papers) & \\
(6 papers) & - Barometric pressure detection & \\
- Brainwave detection (4 papers) & (2 papers) \\
- Heart rate detection (4 papers) & - Fire detection (2 papers) \\
- Head motion detection & - Pothole detection (1 papers) & \\
(3 papers) & & \\
\hline
\end{tabular}

\subsection{Types of Microcontroller Used in Smart Helmets}

Different types of microcontroller have been used to develop smart helmets. By analyzing 103 articles, this study classified the microcontrollers into four categories.

- Chip modular concept unit (MCU): a single computer chip designed for embedded applications (e.g., PIC 18F8720) [110]

- Single-board MCU: a microcontroller built onto a single printed circuit board (e.g., Arduino) [111]

- $\quad$ Single-board computer: a complete computer built on a single circuit board, with microprocessor(s), memory, input/output, and other features required for a functional computer (e.g., Raspberry Pi) [112]

- Smart device: an electronic device, generally connected to other devices or networks by means of different wireless protocols that can operate to some extent interactively and autonomously (e.g., smartphone) [113]

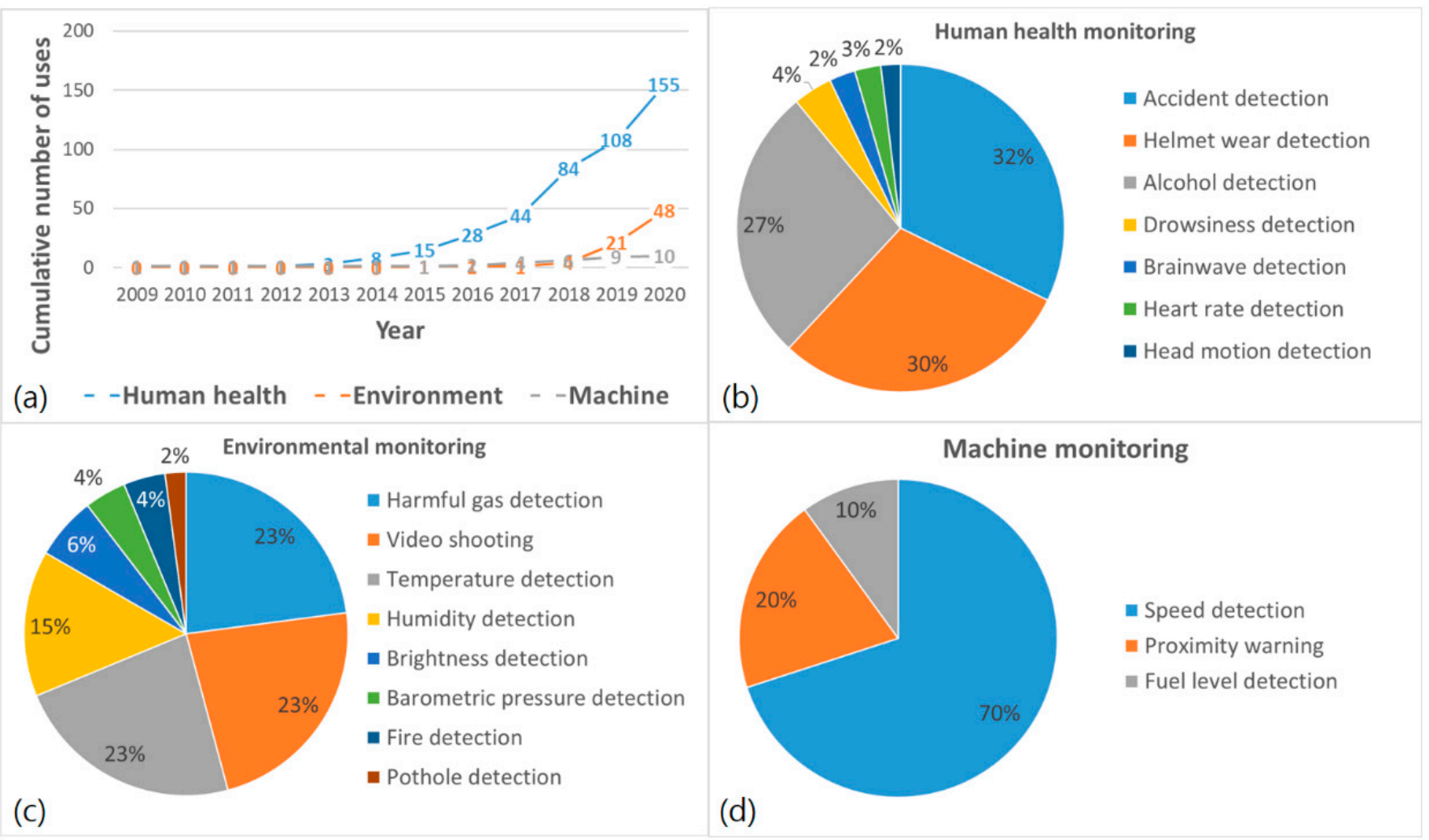

Figure 7. Trend of sensor uses in smart helmet studies. (a) Cumulative number of uses of sensor types according to year. Usage percentage of sensors for (b) human health monitoring, (c) environmental monitoring, and (d) machine monitoring. 
The number of studies that used each of the four types is presented in Figure 8. Among the 103 articles analyzed in this study, the single-board MCU was the most used (56 studies), followed by the chip MCU (28 studies). The number of single-board computers and smart devices used was relatively small (11 and 8 articles, respectively). The use of single-board MCUs has increased rapidly since 2016. Although there have not been many uses of single-board computers so far, their use for smart helmets has increased since 2017. The use of smart devices has been minimal, which is believed to be because of the relatively high cost compared with other devices.

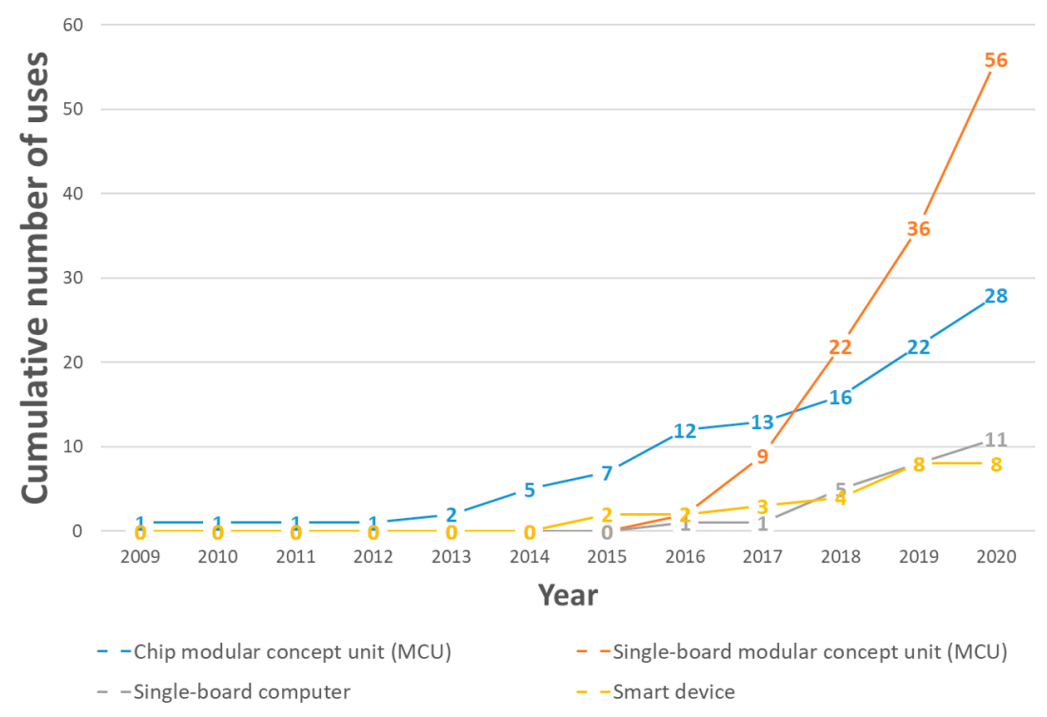

Figure 8. Cumulative number of microcontrollers used in smart helmet studies by year.

Figure 9 shows the use of sensors according to the type of microcontroller in smart helmet studies. Accident, helmet wear, and alcohol detection sensors have been frequently used with the chip MCU and single-board MCU. As shown in Figure 7, the uses of accident, helmet wear, and alcohol detection sensors make up a large part of smart helmet applications. Therefore, it is judged that the uses of chip MCU and single-board MCU were rapidly increased due to the increased development of smart helmets for accident, helmet wear, and alcohol detections. In the case of using a sensor that processes a large amount of data such as video shooting, it can be seen that a single-board computer or a smart device is being used. If the use of sensors based on images or videos increases in smart helmet studies, the single-board computers or smart devices are expected to be used more as microcontrollers.

\subsection{Types of Wireless Communication Technology Used in Smart Helmet Studies}

The data obtained from smart helmets can be transmitted to other smart devices or cloud servers using several wireless communication technologies. Of the 103 articles, 88 reported studies that used wireless communication technology, such as RF, Bluetooth, $\mathrm{Wi}-\mathrm{Fi}$, and Zigbee for this purpose, whereas the remaining did not. Figure 10 shows the cumulative number of uses of wireless communication technology by year for sharing data from smart helmets. According to the analysis results, RF and Bluetooth were the most commonly used, and the number of uses has been increasing rapidly since 2015. Zigbee has been used for the longest time; however, its usage has not increased significantly until recently. 


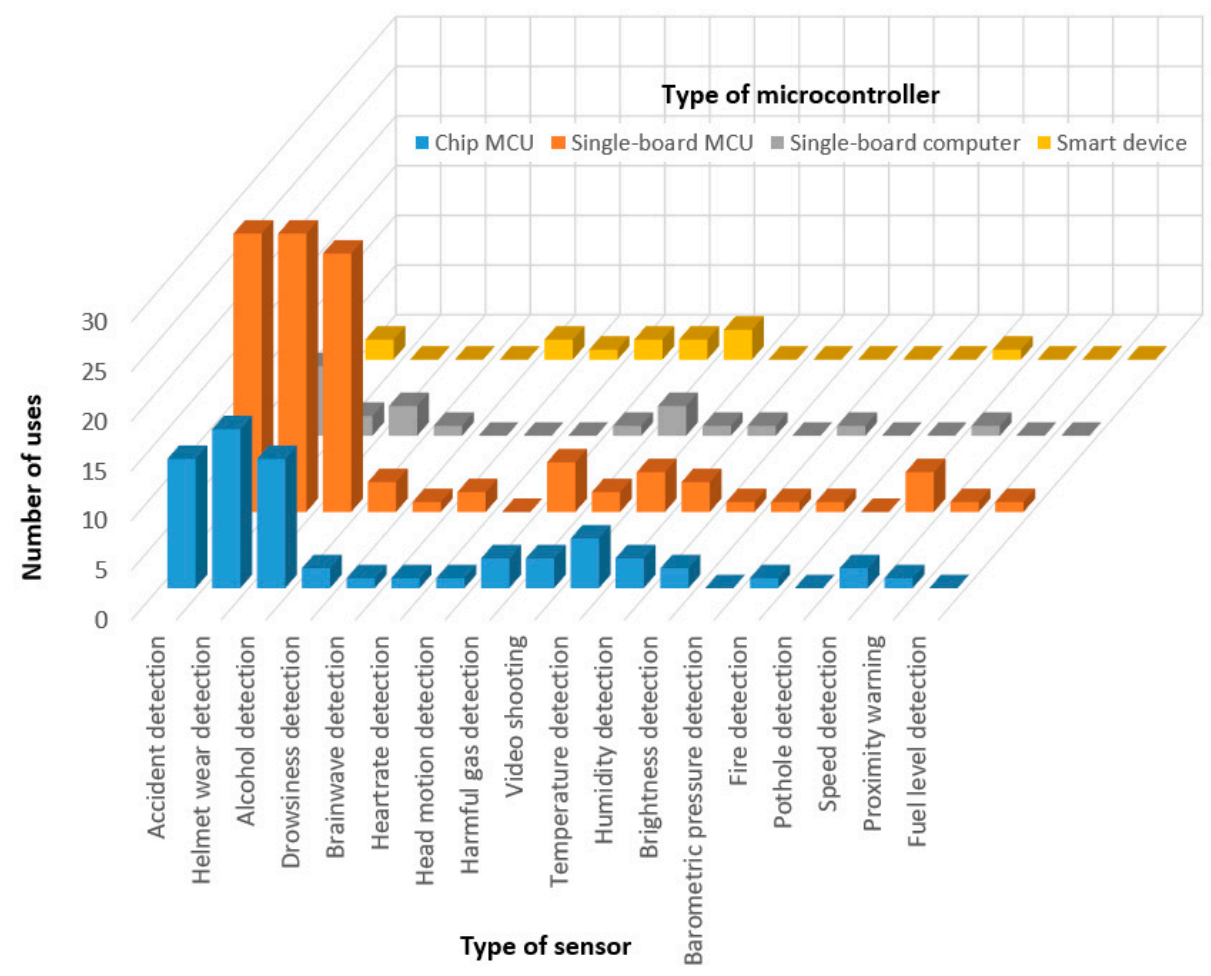

Figure 9. Number of uses by sensor and microcontroller types in smart helmet studies.

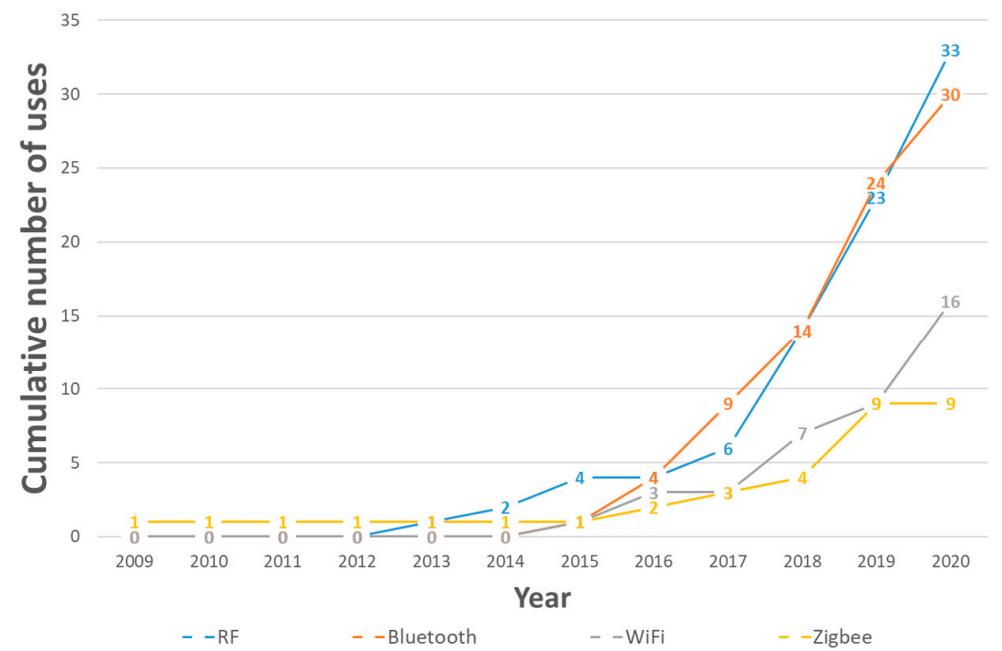

Figure 10. Cumulative number of wireless communication technologies used in smart helmet studies by year.

The reason why RF and Bluetooth are used frequently is related to the microcontroller used in smart helmet applications. Until now, the chip MCU and single-board MCU have been mainly used for smart helmet development, and these two types of microcontrollers often use RF and Bluetooth as shown in Figure 11. In the future, as the use of single-board computers increases, the frequency of use of Wi-Fi is expected to increase. In addition, the uses of Bluetooth and Wi-Fi may increase when the use of smart devices increases in smart helmet studies. 


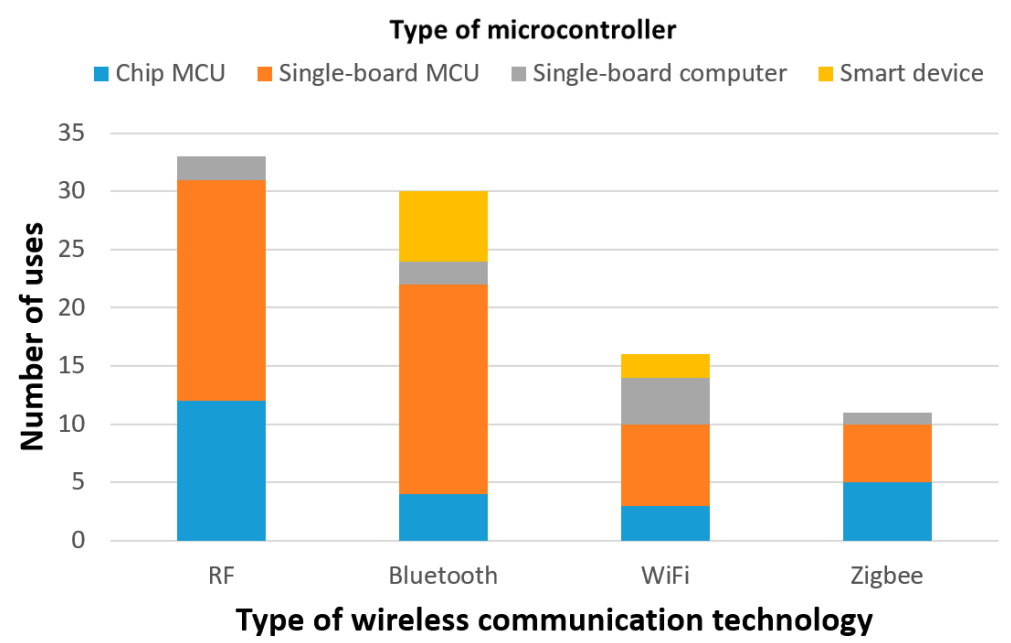

Figure 11. Number of uses by wireless communication technology and microcontroller types in smart helmet studies.

\section{Discussion}

In recent years, smart helmets have rapidly gained popularity among motorcyclists to enhance their safety and comfort. Moreover, safety regulations for workers are being strengthened in many countries; therefore, the growing adoption of advanced wearable technology, including smart helmets, is expected to drive the demand for personal safety. Based on this systematic review, the advantages and limitations of smart helmets can be summarized as follows.

\section{Advantages of smart helmet}

- Smart helmet is an intelligent and reliable wearable device with low costs of development and operation.

- Smart helmets can contribute to improving the safety of motorcyclists and workers by immediately detecting accidents and supporting rescue requests in emergency situations.

- Smart helmets are highly expandable and can be used in other applications by adding or removing sensors as needed.

- Smart helmets can be combined with wireless communication technology to secure the always-on connectivity of a device. Therefore, data can be acquired in real time from sensors attached to the helmet and transmitted to a cloud server for analysis.

\section{Limitations of smart helmet}

- Some sensors, such as cameras and global positioning systems attached to smart helmets have potential elements of privacy invasion. To date, there has been insufficient research on the security and privacy of data collected by smart helmets through sensors.

- Smart helmets mainly use inexpensive and lightweight sensors. Therefore, there is a possibility that the sensor malfunctions frequently, and a false alarm occurs.

- Various sensors can be attached to smart helmets, as needed; however, this can increase the helmet's weight and make it uncomfortable to wear. Moreover, attaching a large battery to power a smart helmet increases the load and can be dangerous to humans. Until now, little research has been conducted on the discomfort and health that people feel when wearing a smart helmet.

For future works, some challenges of smart helmets are as follows.

(1) Power consumption and battery life: As can be seen from the review results, power consumption is expected to increase as various sensors are being added to the smart helmet. Frequent charging and battery replacement can reduce the utility of the smart 
helmet. Therefore, there is a need for a study on how to extend the battery operating time of a smart helmet by using low-power sensors and microcontrollers.

(2) Wearability: Smart helmets are relatively heavy compared to traditional helmets because a variety of sensors and microcontrollers are attached. Motorcyclists or field workers wearing heavy smart helmets may feel uncomfortable. In order for a smart helmet to provide its own functions as a wearable device, it is necessary to reduce the weight of the smart helmet by using lightweight materials and parts. Further research is required for this.

(3) Human health and safety: Because a smart helmet is worn on a person's head, research on the effect of the electromagnetic field emitted from a sensor or microcontroller on human health is required. In addition, it is necessary to consider the safety of the use of smart helmets in places where combustible gases may exist, such as underground mines and chemical facilities.

(4) Durability: Workers wearing smart helmets often work in high humidity and dusty environments. Smart helmets developed to date often have microcontrollers and sensors exposed to the outside of the helmet, and can be vulnerable to high humidity and dust. Research is needed to increase the durability of smart helmets so that smart helmets can provide functions even in harsh working environments.

(5) Accuracy: The sensors attached to the smart helmet should be small in size, lightweight, and low power consumption. Sensors that can meet these conditions at the same time may not have high measurement accuracy. Therefore, there is a need for research on a method that can improve the accuracy of measurement results while satisfying the sensor condition of a smart helmet.

(6) Privacy and data security: The smart helmet collects various information such as photos and videos according to the attached sensors. However, this collection of information can sometimes cause privacy concerns. In addition, there is a possibility of unauthorized users hacking the information in the stage of transmitting the data collected from the smart helmet. Therefore, the privacy and data security issues associated with the use of smart helmets are challenges that require continuous research.

(7) International standards: There are currently no international standards for smart helmets. In order to activate the development and distribution of smart helmets, it is necessary to consult and finance international standards for smart helmet specifications, functions, sensors, microcontrollers, wireless communication technology, etc.

\section{Conclusions}

A systematic review was conducted to investigate the current status and trends of smart helmet studies published in the past 11 years (2009-2020). The review provided answers to five RQs, as follows.

(1) The number of studies on smart helmets has increased rapidly since 2018. In the last three years (2018-2020), these studies accounted for $74.8 \%$ of the total number of studies. Therefore, it can be said that the research on smart helmets has been receiving considerable attention, and it is expected to be expanded further in the future.

(2) Studies on smart helmets can be divided into two categories (i.e., motorcyclists and workers) depending on the user. Among these, the application of smart helmets to motorcyclists accounts for $79 \%$. Because helmets are necessary items that should be worn by motorcyclists, smart helmet studies have attracted relatively more attention in this field. Most smart helmets for motorcyclists provide functions for starting a motorcycle and rescue requests in emergency situations. To date, research on smart helmets for workers has been inadequate.

(3) Among the various sensors used for developing smart helmets, those related to human health monitoring have been used the most, and the last three years (2018-2020) have seen the highest increase. Among them, sensors for detecting accidents, helmet wearing status, and alcohol were frequently used, accounting for $32 \%, 30 \%$, and $27 \%$, respectively. Note that the use of environmental monitoring sensors for harmful gas 
detection, image information acquisition, and temperature measurement, has rapidly increased since 2019.

(4) Currently, the type of microcontroller primarily used for developing smart helmets is a single-board MCU, such as the Arduino board, which accounts for more than 50\% of all microcontroller types. Although single-board computers such as the Raspberry Pi or smartphones are also being used to develop smart helmets, this number has not been so far.

(5) To date, approximately $85 \%$ of smart helmets have been developed using wireless communication technologies, such as RF, Bluetooth, WiFi, and Zigbee. Among them, the use of RF and Bluetooth has been increasing rapidly since 2015, and they are the most commonly used technologies today, accounting for $38 \%$ and $34 \%$, respectively.

Considering the expandability and functionality of smart helmets, they can be widely used not only for various sporting events but also for military purposes. However, until now, few cases of such applications have been reported in academic papers. Academic studies on smart helmets are needed to expand their application to more diverse fields, including sports and military services. Therefore, the findings of this study are expected to benefit researchers and industry professionals in various fields by offering detailed and systematic analysis results of smart helmet technologies to the community, thereby bridging the gap between academia and industry. In addition, it can help practitioners recognize the potential applications of smart helmets and understand the requirements and challenges of smart helmet products in various fields.

Author Contributions: Conceptualization, Y.C.; methodology, Y.C.; software, Y.C.; validation, Y.K.; formal analysis, Y.K.; investigation, Y.C.; resources, Y.C.; data curation, Y.K.; writing-original draft preparation, Y.C. and Y.K.; writing-review and editing, Y.C.; visualization, Y.K.; supervision, Y.C.; project administration, Y.C.; funding acquisition, Y.C. All authors have read and agreed to the published version of the manuscript.

Funding: This work was supported by a National Research Foundation of Korea (NRF) grant funded by the Korean government (MSIT) (2021R1A2C1011216).

Institutional Review Board Statement: Not applicable.

Informed Consent Statement: Not applicable.

Data Availability Statement: Data sharing not applicable.

Conflicts of Interest: The authors declare no conflict of interest.

\section{References}

1. Wrzesińska, N. The use of smart glasses in healthcare-Review. MEDtube Sci. 2015, 3, 31-34.

2. Choi, B.; Hwang, S.; Lee, S.H. What drives construction workers' acceptance of wearable technologies in the workplace? Indoor localization and wearable health devices for occupational safety and health. Autom. Constr. 2017, 84, 31-41. [CrossRef]

3. Audi Uses Wearables in Logistics. Available online: https://www.volkswagenag.com/en/news/2016/11/Audi_Wearables.html (accessed on 5 February 2018).

4. TECHZONE360. Available online: http://www.techzone360.com/topics/techzone/articles/2016/06/27/422510-6-insurancecompanies-investing-wearable-technology.htm\# (accessed on 20 November 2017).

5. Wright, R.; Keith, L. Wearable Technology: If the Tech Fits, Wear It. J. Electron. Resour. Med Libr. 2014, 11, 204-216. [CrossRef]

6. Grand View Research. Available online: https://www.grandviewresearch.com/industry-analysis/smarthelmet-market (accessed on 7 October 2020).

7. Singh, V.; Chandna, H.; Upadhyay, N. SmartPPM: An Internet of Things Based Smart helmet Design for Potholes and Air Pollution Monitoring. EAI Endorsed Trans. Internet Things 2019, 5, 1-9. [CrossRef]

8. Wang, C.; Kim, Y.; Kim, D.G.; Lee, S.H.; Min, S.D. Smart helmet and Insole Sensors for Near Fall Incidence Recognition during Descent of Stairs. Appl. Sci. 2020, 10, 2262. [CrossRef]

9. Mohammed, M.N.; Syamsudin, H.; Al-Zubaidi, S.; Yusuf, E. Novel Covid-19 Detection and Diagnosis System Using Iot Based Smart Helmet. Int. J. Psychosoc. Rehabil. 2020, 24, 2296-2303.

10. Rasli, M.K.A.; Madzhi, N.K.; Johari, J. Smart Helmet with Sensors for Accident Prevention. In Proceedings of the 2013 International Conference on Electrical, Electronics and System Engineering (ICEESE), Kuala Lumpur, Malaysia, 4-5 December 2013; pp. 21-26. [CrossRef] 
11. Vijayan, S.; Govind, V.T.; Mathews, M.; Surendran, S. Alcohol Detection Using Smart Helmet System. Int. J. Emerg. Technol. Comput. Sci. Electron. 2014, 8, 190-195.

12. Agarwal, N.; Singh, A.K.; Singh, P.P.; Sahani, R. Smart helmet. Int. Res. J. Eng. Technol. 2015, 2, $19-22$.

13. Gautam, A.S.; Dubey, G.K.; Mishra, M.; Prabhat, M. Smart helmet System. J. Emerg. Technol. Innov. Res. 2015, 2, 1165-1168.

14. Melcher, V.; Diederichs, F.; Maestre, R.; Hofmann, C.; Nacenta, J.-M.; van Gent, J.; Kusić, D.; Žagar, B. Smart Vital Signs and Accident Monitoring System for Motorcyclists Embedded in Helmets and Garments for Advanced ECall Emergency Assistance and Health Analysis Monitoring. Procedia Manuf. 2015, 3, 3208-3213. [CrossRef]

15. Chandran, S.; Chandrasekar, S.; Elizabeth, N.E. Konnect: An Internet of Things (IoT) Based Smart helmet for Accident Detection and Notification. In Proceedings of the 2016 IEEE Annual India Conference (INDICON), Bangalore, India, 16-18 December 2016; pp. 1-4. [CrossRef]

16. Magno, M.; D’Aloia, A.; Polonelli, T.; Spadaro, L.; Benini, L. SHelmet: An Intelligent Self-Sustaining Multi Sensors Smart helmet for Bikers. In Sensor Systems and Software; Lecture Notes of the Institute for Computer Sciences, Social Informatics and Telecommunications Engineering; Magno, M., Ferrero, F., Bilas, V., Eds.; Springer International Publishing: Cham, Switzerland, 2017; Volume 205, pp. 55-67. [CrossRef]

17. Nikharge, B.J.; Poojary, M.M.; Pooja, T. Smart Helmet-Intelligent Safety for Motorcyclist Using Raspberry Pi and Open Cv. Int. Res. J. Eng. Technol. 2016, 3, 589-593.

18. Jadhawar, M.; Kandepalli, G.; Kohade, A.; Komati, R. Smart Helmet Safety System Using Atmega 32. Int. J. Res. Eng. Technol. 2016, 5, 287-289.

19. Kim, J.; Koo, S.E.; Lim, J.Y.; Jin, M.W.; Choi, J.M. Smart Helmet. In Proceedings of the ICCC 2016 International Conference on Convergence Content, Kunsan, Korea, 22-27 May 2016; pp. 357-358.

20. Kumar, S.; Srikrishnan, A.; Ravi, S. Cloud Incorporated Smart helmet Integrated with Two-Wheeler Communication Setup. Int. J. Control. Theory Appl. 2016, 9, 251-261.

21. Vashisth, R.; Gupta, S.; Jain, A.; Gupta, S.; Sahil; Rana, P. Implementation and Analysis of Smart helmet. In Proceedings of the 2017 4th International Conference on Signal Processing, Computing and Control (ISPCC), Solan, India, 21-23 September 2017; pp. 111-117. [CrossRef]

22. Varade, A.; Gajbhiye, N.; Panchbhai, A.V.V. Smart Helmet Using GSM and GPS. Int. Res. J. Eng. Technol. 2017, 04, 1662-1667.

23. Rajput, A.; Saxena, A.; Agarwal, A.; Bhatia, A.; Mishra, A. Smart helmet with Rider Safety System. Int. J. Innov. Emerg. Res. Eng. 2017, 4, 52-60.

24. Patel, M.V.A.; Mishra, M.A.; Hiten, M.R.; Prajapati, M.K. Smart helmet. Int. Res. J. Eng. Technol. 2017, 4, 7-10.

25. Muthiah, M.; Aswin Natesh, V.; Sathiendran, R.K. Smart helmets for Automatic Control of Headlamps. In Proceedings of the 2015 International Conference on Smart Sensors and Systems (IC-SSS), Bangalore, India, 21-23 December 2015; pp. 1-4. [CrossRef]

26. Ahuja, P.; Bhavsar, K. Microcontroller Based Smart helmet Using GSM \& GPRS. In Proceedings of the 2018 2nd International Conference on Trends in Electronics and Informatics (ICOEI), Tirunelveli, India, 11-12 May 2018; pp. 1-9. [CrossRef]

27. Godwani, P.D.; Dhokrat, A.U.; Kubde, G.D.; Kumbhar, R.R.; Mahajan, R.G. Smart helmet for Accident Detection and Navigation. Int. J. Creat. Res. Thoughts 2018, 6, 788-792.

28. Parameshwari, P.; Pujari, V.; Gadgay, B. Smart Helmet for Accident Prevention. Int. Res. J. Eng. Technol. 2018, 5, 368-370.

29. Biswas, J.R.; Kachroo, S.; Chopra, P.; Sharma, S. Development of an App Enabled Smart helmet for Real Time Detection and Reporting of Accidents. In Proceedings of the 2018 7th International Conference on Reliability, Infocom Technologies and Optimization (Trends and Future Directions (ICRITO), Noida, India, 29-31 August 2018; pp. 703-708. [CrossRef]

30. Budiman, A.R.; Sudiharto, D.W.; Brotoharsono, T. The Prototype of Smart helmet with Safety Riding Notification for Motorcycle Rider. In Proceedings of the 2018 3rd International Conference on Information Technology, Information System and Electrical Engineering (ICITISEE), Yogyakarta, Indonesia, 13-14 November 2018; pp. 362-367. [CrossRef]

31. Uniyal, M.; Rawat, H.; Srivastava, M.; Srivastava, V.K. IOT Based Smart helmet System with Data Log System. In Proceedings of the 2018 International Conference on Advances in Computing, Communication Control and Networking (ICACCCN), Greater Noida, India, 12-13 October 2018; pp. 28-31. [CrossRef]

32. Paulchamy, D.B.; Sundhararajan, C.; Xavier, R.; Ramkumar, A.; Vigneshwar, D. Design of Smart helmet and Bike Management System. Asian J. Appl. Sci. Technol. 2018, 2, 207-211.

33. Souza, A.D.; Maliyackal, S.S. Helmet Integrated Bike Ignition Using Arduino. In Proceedings of the 4th International Conference on Energy Efficient Technologies for Sustainability-ICEETS'18, Tamil Nadu, India, 5-7 April 2018; pp. 1-5.

34. Nanda, S.; Joshi, H.; Khairnar, S. An IOT Based Smart System for Accident Prevention and Detection. In Proceedings of the 2018 Fourth International Conference on Computing Communication Control and Automation (ICCUBEA), Pune, India, 16-18 August 2018; pp. 1-6. [CrossRef]

35. Deva Kumar, S.V.S.V.P.; Akashe, S.; Kumar, V. Advanced Control of Switching Ignition by Smart helmet. Int. J. Image Graph. Signal Process. 2018, 10, 34-42. [CrossRef]

36. Sumamah, M.; Faiz, M.; Tyagi, A. Smart helmet kit. Int. Res. J. Eng. Technol. 2018, 5, 3018-3020.

37. Shabbeer, S.A.; Meleet, M. Smart helmet for Accident Detection and Notification. In Proceedings of the $20172 \mathrm{nd}$ International Conference on Computational Systems and Information Technology for Sustainable Solution (CSITSS), Bengaluru, India, 21-23 December 2017; pp. 1-5. [CrossRef] 
38. Gudavalli, D.K.P.; Rani, B.S.; Sagar, C.V. Helmet Operated Smart E-Bike. In Proceedings of the 2017 IEEE International Conference on Intelligent Techniques in Control, Optimization and Signal Processing (INCOS), Srivilliputhur, India, 23-25 March 2017; pp. 1-5. [CrossRef]

39. Tapadar, S.; Ray, S.; Saha, H.N.; Saha, A.K.; Karlose, R. Accident and Alcohol Detection in Bluetooth Enabled Smart helmets for Motorbikes. In Proceedings of the 2018 IEEE 8th Annual Computing and Communication Workshop and Conference (CCWC), Las Vegas, NV, USA, 8-10 January 2018; pp. 584-590. [CrossRef]

40. Premalatha, K.; Nandhini, J.J. Safeguarding Two Wheeler User's Lives Using Smart helmet. Int. J. Innov. Technol. Explor. Eng. 2018, 8, 417-419.

41. Dhulavvagol, P.M.; Shet, R.; Nashipudi, P.; Meti, A.S.; Ganiger, R. Smart helmet with Cloud GPS GSM Technology for Accident and Alcohol Detection. In Cognitive Computing and Information Processing; Communications in Computer and Information Science; Nagabhushan, T.N., Aradhya, V.N.M., Jagadeesh, P., Shukla, S.M.L.C., Eds.; Springer: Singapore, 2018; Volume 801, pp. 346-357. [CrossRef]

42. Joshi, S.B.; Joshi, A.S. A Novel Method for Safety of Two Wheeler Using Microcontroller. Int. J. Adv. Res. Innov. Ideas Educ. 2019, 5, 1439-1442.

43. Namayala, P.P. Development of Smart helmet Motor Cycles' Embedded System. Int. J. Eng. Res. Adv. Technol. 2019, 5, 75-84. [CrossRef]

44. Wong, K.I.; Chen, Y.-C.; Lee, T.-C.; Wang, S.-M. Head Motion Recognition Using a Smart helmet for Motorcycle Riders. In Proceedings of the 2019 International Conference on Machine Learning and Cybernetics (ICMLC), Kobe, Japan, 7-10 July 2019; pp. 1-7. [CrossRef]

45. Kinage, V.; Patil, P. IoT Based Intelligent System for Vehicle Accident Prevention And Detection At Real Time. In Proceedings of the 2019 IoThird International conference on I-SMACT in Social, Mobile, Analytics and Cloud (I-SMAC), Palladam, India, 12-14 December 2019; pp. 409-413. [CrossRef]

46. Swathi, S.J.; Raj, S.; Devaraj, D. Microcontroller and Sensor Based Smart Biking System for Driver's Safety. In Proceedings of the 2019 IEEE International Conference on Intelligent Techniques in Control, Optimization and Signal Processing (INCOS), Tamilnadu, India, 11-13 April 2019; pp. 1-5. [CrossRef]

47. Reddy, D.D.V.; Suresh, V.; Hemalatha, T. Smart helmet and Bike Management System. J. Gujarat Res. Soc. $2019,21,303-311$.

48. Shravya, K.; Mandapati, Y.; Keerthi, D.; Harika, K.; Senapati, R.K. Smart helmet for Safe Driving. E3S Web Conf. 2019, 87, 1-4. [CrossRef]

49. Kanimozhi, L.; Sambasivam, R.; Pragathi, M.; Ranjith, M. Smart helmet with Accident Avoidance System. Cikitusi J. Multidiscip. Res. 2019, 6, 1-7.

50. Patil, M.S.A.; Wagh, M.V.R.; Ganpatre, M.V.J. IOT Based Smart helmet For Accident Detection. Resincap J. Sci. Eng. 2019, 3, $455-458$.

51. Jesudoss, A.; Vybhavi, R.; Anusha, B. Design of Smart helmet for Accident Avoidance. In Proceedings of the 2019 International Conference on Communication and Signal Processing (ICCSP), Chennai, India, 4-6 April 2019; pp. 0774-0778. [CrossRef]

52. Kabilan, M.; Monish, S.; Siamala Devi, D.S. Accident Detection System Based on Internet of Things (IoT)-Smart helmet. Int. J. Eng. Res. Adv. Technol. 2019, 5, 154-157.

53. Ashwin, M.; Yashwanth Gowda, S. Smart helmet Using GPS and GSM Modem. Int. J. Eng. Res. Adv. Technol. 2019, 8, 3005-3008. [CrossRef]

54. Chen, Y.-R.; Tsai, C.-M.; Wong, K.-I.; Lee, T.-C.; Loh, C.-H.; Ying, J.-C.; Chen, Y.-C. Motorcyclists' Head Motions Recognition by Using the Smart helmet with Low Sampling Rate. In Proceedings of the 2019 Twelfth International Conference on Ubi-Media Computing (Ubi-Media), Bali, Indonesia, 5-8 August 2019; pp. 157-163. [CrossRef]

55. Gupta, S.; Sharma, K.; Salvekar, N.; Gajra, A. Implementation of Alcohol and Collision Sensors in a Smart helmet. In Proceedings of the 2019 Conception of smart iol helmet in smart factory (ICNTE), Navi Mumbai, India, 4-5 January 2019; pp. 1-5. [CrossRef]

56. Priya, V.; Dhanasekar, J.; Vasumathi, G. Smart helmet Using PIC Controller. Int. J. Eng. Res. Adv. Technol. 2019, 8, 750-752. [CrossRef]

57. Vijayakumar, D.; Ramesh, G.; Jayabalan, C.; Palani, S.; Selvam, M. Micro Controller Based Smart helmet by Ir Motion Sensors. Int. J. Eng. Res. Adv. Technol. 2019, 8, 1850-1853. [CrossRef]

58. Divyasudha, N.; Arulmozhivarman, P.; Rajkmumar, E.R. Analysis of Smart helmets and Designing an IoT Based Smart helmet: A Cost Effective Solution for Riders. In Proceedings of the 2019 1st International Conference on Innovations in Information and Communication Technology (ICIICT), Chennai, India, 25-26 April 2019; pp. 1-4. [CrossRef]

59. Mhatre, K.; Nandwadekar, R.; Patil, A.; Shinde, R.; Kamble, P. Smart helmet With Intercom Feature. SSRN J. 2020, 1-5. [CrossRef]

60. Lakshmanachari, S.; Ramya, M. Smart helmet System for Identification of Road Accident Using Internet of Things. Int. J. Adv. Sci. Technol. 2020, 29, 1070-1076.

61. Merlin, R.; Pranay, R.D. Smart helmet system. TEST Eng. Manag. 2020, 83, 1991-1995.

62. Chidambarathanu, G.V.; Farzana, D.F.; Gowrishankar, J. Accidents Preventing Smart helmet Using EEG Sensor. TEST Eng. Manag. 2020, 82, 2541-2547.

63. Santhanakrishnan, C.; Sharma, D.; Vashistha, A. Smart helmet for Rider (SHR) and Accident Detection Using IOT. Int. J. Adv. Sci. Technol. 2020, 29, 50-57. 
64. Sai Kumar, M.; Aruna, M. Third Eye Two Wheeler: Accident and Malt Detection in Bluetooth Enabled Smart helmets with Load Monitoring for Motorbikes. TEST Eng. Manag. 2020, 82, 6696-6701.

65. Suman, A.; Parashar, A.; Shukla, A.; Shobha, K.R. Aagaahi-A Smart helmet. In Proceedings of the 2020 IEEE International Conference on Electronics, Computing and Communication Technologies (CONECCT), Bangalore, India, 2-4 July 2020; pp. 1-6. [CrossRef]

66. Ahmed, S.U.; Uddin, R.; Affan, M. Intelligent Gadget for Accident Prevention: Smart helmet. In Proceedings of the 2020 International Conference on Computing and Information Technology (ICCIT-1441), Tabuk, Saudi Arabia, 9-10 September 2020; pp. 1-4. [CrossRef]

67. Dubey, S.; Meghana, K.; Likhitha, M.; Gupta, K.; Balaji, R. An Experimental Study on Advanced Lane Changing Signal Assist Technology with Smart helmet. Mater. Today Proc. 2020, 33, 4771-4776. [CrossRef]

68. Lokeshwaran, M.; Nikhit Mathew, S.P.; Joshuva, A. Raphael-The Smart helmet. In Proceedings of the 2020 International Conference on Wireless Comunications Signal Processing and Networking (WiSPNET), Chennai, India, 19-21 March 2020; pp. $48-51$.

69. Rahman, M.A.; Ahsanuzzaman, S.M.; Rahman, I.; Ahmed, T.; Ahsan, A. IoT Based Smart helmet and Accident Identification System. In Proceedings of the 2020 IEEE Region 10 Symposium (TENSYMP), Dhaka, Bangladesh, 5-7 June 2020; pp. 14-17. [CrossRef]

70. Faikul, U.; Hairil, B.; Ach, D. Smart helmet Control System Using Heart Pulse Indicator. In Proceedings of the 2020 International Conference on Science and Technology, Surabaya, Indonesia, 3 November 2020; pp. 1-8.

71. Rao, P.K.; Sai, P.T.; Kumar, N.V.; Sagar, S.Y.V. Design and Implementation of Smart helmet Using IoT. In Proceedings of the 2020 International Confernce of Advance Research and Innovation, Meerut, India, 19 January 2020; pp. 323-325.

72. Oviyaa, M.; Renvitha, P.; Swathika, R.; Paul, I.J.L.; Sasirekha, S. Arduino Based Real Time Drowsiness and Fatigue Detection for Bikers Using Helmet. In Proceedings of the 2020 2nd International Conference on Innovative Mechanisms for Industry Applications (ICIMIA), Bangalore, India, 5-7 March 2020; pp. 573-577. [CrossRef]

73. Shahare, B.; Chawde, S.; Gudafwar, R.; Pal, H.; Bobade, P. Iot Based Smart Motor Cycle Helmet. Int. J. Progress. Res. Sci. Eng. 2020, 1, 107-109.

74. Ashwini, S.S.; Udupa, N.G.; Sweta, N.; Alam, M.S. A Smart Helmet on IoT Technology for Safety and Accident Detection. Int. J. R D Eng. Sci. Manag. 2020, 3, 99-102.

75. Jayasinghe, S.; Arachchige, U. A Smart helmet with a built-in drowsiness and alcohol detection system. J. Res. Technol. Eng. 2020, 1, 76-81.

76. Aravinda, N.L.; Jabirullah, M.; DubasiKirtana. An Intelligent Helmet System Using IoT and Raspberry Pi. IOP Conf. Ser. Mater. Sci. Eng. 2020, 981, 1-7. [CrossRef]

77. MohanaRoopa, D.Y.; Soujanya, N.; Vaishnavi, V.S.; Vardhan, U.V. An IOT Based Smart helmet for Accident Detection and Notification. J. Interdiscip. Cycle Res. 2020, 7, 1-7.

78. Pothirajan, M.G.; Mary, M.J.V. IOT Based Vehicle Monitoring Using Smart Helmet. Alochana Chakra J. 2020, 15, 671-674.

79. Swarna, V.; Deepika, P.; Zaheer, S.; Raj, S.M.; Rao, E.K. Smart helmet for Rider's Safety. J. Res. Sci. Eng. Manag. $2020,6,13-17$.

80. Parakkal, S.A.; Avhad, P.V.; Dhole, V.; Raikar, Y.; Gite, B.B. Smartphone Integrated Smart helmet for Real-Time Detection, Prevention and Reporting of Accidents. Bull. Monum. 2020, 21, 41-45.

81. Kumar Kar, S.; Anshuman, D.A.; Raj, H.; Pall Singh, P. New Design and Fabrication of Smart helmet. IOP Conf. Ser. Mater. Sci. Eng. 2018, 402, 1-10. [CrossRef]

82. Rahman, A.; Abdurohman, M.; Putrada, A.G. Indicator Warning Refined Fuel Oil in A Motorcycle With Fuzzy Logic and Sound Navigaiotn Through Smart helmet. In Proceedings of the 2019 International Symposium on Electronics and Smart Devices (ISESD), Badung, Indonesia, 8-9 October 2019; pp. 1-5. [CrossRef]

83. Jadhav, A.; Rajput, S.; Baburao, K.V.; Rajput, D.D.S. Smart helmet Using Natural Language Processing, Head Mounted Display and Solar Panel. Int. J. Sci. Technol. Res. 2019, 8, 331-527.

84. Youssef, A.; Colon, J.; Mantzios, K.; Gkiata, P.; Mayor, T.; Flouris, A.; De Bruyne, G.; Aerts, J.-M. Towards Model-Based Online Monitoring of Cyclist's Head Thermal Comfort: Smart helmet Concept and Prototype. Appl. Sci. 2019, 9, 3170. [CrossRef]

85. Rao, S. Voice Controlled Wiper for Smart helmets. Int. J. Innov. Technol. Explor. Eng. 2019, 8, 2086-2089. [CrossRef]

86. Kanetkar, S.; Rathore, A.; Maheshwari, K.; Dubey, P.; Saxena, A. Smart helmet Wiper. In Proceedings of the 2020 IEEE International Students' Conference on Electrical, Electronics and Computer Science (SCEECS), Bhopal, India, 22-23 February 2020; pp. 1-4. [CrossRef]

87. Aatif, M.K.A.; Manoj, A. Smart helmet Based on IoT Technology. Int. J. Res. Appl. Sci. Eng. Technol. 2017, 5, 409-413.

88. Ajay, A.; Vishnu, G.; Kishoreswaminathan, V.; Vishwanth, V.; Srinivasan, K.; Jeevanantham, S. Accidental Identification and Navigation System in Helmet. In Proceedings of the 2017 International Conference on Nextgen Electronic Technologies: Silicon to Software (ICNETS2), Chennai, India, 23-25 March 2017; pp. 202-204. [CrossRef]

89. Kumar, A.; Manjunath, S.S.; Monish, R.; Ramya, S. The Intercom Enabled Helmet. Int. Res. J. Eng. Technol. 2019,6 , $3986-3988$.

90. Qiang, C.; Ji-ping, S.; Zhe, Z.; Fan, Z. ZigBee Based Intelligent Helmet for Coal Miners. In Proceedings of the 2009 WRI World Congress on Computer Science and Information Engineering, Los Angeles, CA, USA, 31 March-2 April 2009; pp. $433-435$. [CrossRef] 
91. Shabina, S. Smart helmet Using RF and WSN Technology for Underground Mines Safety. In Proceedings of the 2014 International Conference on Intelligent Computing Applications, Coimbatore, India, 6-7 March 2014; pp. 305-309. [CrossRef]

92. Behr, C.J.; Kumar, A.; Hancke, G.P. A Smart helmet for Air Quality and Hazardous Event Detection for the Mining Industry. In Proceedings of the 2016 IEEE International Conference on Industrial Technology (ICIT), Taipei, Taiwan, 14-17 March 2016; pp. 2026-2031. [CrossRef]

93. Hazarika, P. Implementation of Smart Safety Helmet for Coal Mine Workers. In Proceedings of the 2016 IEEE 1st International Conference on Power Electronics, Intelligent Control and Energy Systems (ICPEICES), Delhi, India, 4-6 July 2016; pp. 1-3. [CrossRef]

94. Sharma, M.; Maity, T. Low Cost Low Power Smart helmet for Real-Time Remote Underground Mine Environment Monitoring. Wirel. Pers Commun. 2018, 102, 149-162. [CrossRef]

95. Revindran, R.; Vijayaraghavan, H.; Huang, M.-Y. Smart helmets for Safety in Mining Industry. In Proceedings of the 2018 International Conference on Advances in Computing, Communications and Informatics (ICACCI), Bangalore, India, 19-22 September 2018; pp. 217-221. [CrossRef]

96. Eldemerdash, T.; Abdulla, R.; Jayapal, V.; Nataraj, C.; Abbas, K. Iot Based Smart helmet for Mining Industry Application. Int. J. Adv. Sci. Technol. 2020, 29, 373-387.

97. Sanjay, B.S.; Dilip, K.A.; Balasaheb, T.A.; KinnuKumar, S.; Saware, N.P. Smart helmet Using Zigbee. Int. J. Innov. Res. Technol. 2019, 6, 144-148.

98. Charde, A.; Dehankar, B.; Ghaturle, S.; Bende, B.; Kitey, S. A Smart and Secured Helmet for Coal Mining Workers. J. Res. Appl. Sci. Eng. Technol. 2020, 8, 673-675. [CrossRef]

99. Sujitha, S.; Loret, S.; Gethsy, M. IOT Based Smart Mine Safety System Using Arduino. Int. J. Comput. Sci. Mob. Comput. 2020, 9 , 141-145.

100. Pirkl, G.; Hevesi, P.; Amarislanov, O.; Lukowicz, P. Smart helmet for Construction Site Documentation and Work Support. In Proceedings of the 2016 ACM International Joint Conference on Pervasive and Ubiquitous Computing, Heidelberg, Germany, 12-16 September 2016; pp. 349-352. [CrossRef]

101. Lee, A.; Moon, J.; Min, S.D.; Sung, N.-J.; Hong, M. Safety Analysis System Using Smart helmet. Internet Comput. Internet Things 2019, 503, 102-107.

102. Li, P.; Meziane, R.; Otis, M.J.-D.; Ezzaidi, H.; Cardou, P. A Smart Safety Helmet Using IMU and EEG Sensors for Worker Fatigue Detection. In Proceedings of the 2014 IEEE International Symposium on Robotic and Sensors Environments (ROSE), Timisoara, Romania, 16-18 October 2014; pp. 55-60. [CrossRef]

103. Dhingra, J.; Arora, G.; Bhatia, P.; Goel, C. Smart helmet with Inter-Communication System, Smart Reminder System and Panic Button for Emergencies. Int. J. Eng. Res. 2018, 7, 319-320.

104. Aston, J.P.; Benko, N.; Truong, T.; Zaki, A.; Olsen, N.; Eshete, E.; Luttmer, N.G.; Coats, B.; Minor, M.A. Optimization of a Soft Robotic Bladder Array for Dissipating High Impact Loads: An Initial Study in Designing a Smart helmet. In Proceedings of the 2020 3rd IEEE International Conference on Soft Robotics (RoboSoft), New Haven, CT, USA, 15 May-15 July 2020; pp. 607-614. [CrossRef]

105. Campero-Jurado, I.; Márquez-Sánchez, S.; Quintanar-Gómez, J.; Rodríguez, S.; Corchado, J.M. Smart helmet 5.0 for Industrial Internet of Things Using Artificial Intelligence. Sensors 2020, 20, 6241. [CrossRef] [PubMed]

106. Shu, L.; Li, K.; Zen, J.; Li, X.; Sun, H.; Huo, Z.; Han, G. A Smart helmet for Network Level Early Warning in Large Scale Petrochemical Plants. In Proceedings of the 14th International Conference on Information Processing in Sensor Networks-IPSN '15; ACM Press: Seattle, WA, USA, 2015; pp. 390-391. [CrossRef]

107. Bisio, I.; Fedeli, A.; Lavagetto, F.; Pastorino, M.; Randazzo, A.; Sciarrone, A.; Tavanti, E. Mobile Smart helmet for Brain Stroke Early Detection through Neural Network-Based Signals Analysis. In Proceedings of the GLOBECOM 2017-2017 IEEE Global Communications Conference, Singapore, 4-8 December 2017; pp. 1-6. [CrossRef]

108. Shahiduzzaman, K.M.; Hei, X.; Guo, C.; Cheng, W. Enhancing Fall Detection for Elderly with Smart helmet in a Cloud-NetworkEdge Architecture. In Proceedings of the 2019 IEEE International Conference on Consumer Electronics-Taiwan (ICCE-TW), Yilan, Taiwan, 20-22 May 2019; pp. 1-2. [CrossRef]

109. Jeong, M.; Lee, H.; Bae, M.; Shin, D.-B.; Lim, S.-H.; Lee, K.B. Development and Application of the Smart helmet for Disaster and Safety. In Proceedings of the 2018 International Conference on Information and Communication Technology Convergence (ICTC), Jeju, Korea, 17-19 October 2018; pp. 1084-1089. [CrossRef]

110. Wikipedia. Microcontroller. Available online: https://en.wikipedia.org/wiki/Microcontroller (accessed on 25 February 2021).

111. Wikipedia. Single-Board Microcontroller. Available online: https://en.wikipedia.org/wiki/Single-board_microcontroller (accessed on 25 February 2021).

112. Wikipedia. Single-Board Computer. Available online: https://en.wikipedia.org/wiki/Single-board_computer\#Types,_standards (accessed on 25 February 2021).

113. Wikipedia. Smart Device. Available online: https://en.wikipedia.org/wiki/Smart_device (accessed on 25 February 2021). 